ARTICLE

DOI: 10.1038/ncomms11298

OPEN

\title{
Myelinating satellite oligodendrocytes are integrated in a glial syncytium constraining neuronal high-frequency activity
}

\author{
Arne Battefeld ${ }^{1}$, Jan Klooster ${ }^{1} \&$ Maarten H.P. Kole ${ }^{1,2}$
}

Satellite oligodendrocytes (s-OLs) are closely apposed to the soma of neocortical layer 5 pyramidal neurons but their properties and functional roles remain unresolved. Here we show that $\mathrm{s}$-OLs form compact myelin and action potentials of the host neuron evoke precisely timed $\mathrm{Ba}^{2+}$-sensitive $\mathrm{K}^{+}$inward rectifying (Kir) currents in the s-OL. Unexpectedly, the glial $\mathrm{K}^{+}$inward current does not require oligodendrocytic Kir4.1. Action potential-evoked Kir currents are in part mediated by gap-junction coupling with neighbouring OLs and astrocytes that form a syncytium around the pyramidal cell body. Computational modelling predicts that glial Kir constrains the perisomatic $\left[\mathrm{K}^{+}\right]_{\mathrm{o}}$ increase most importantly during high-frequency action potentials. Consistent with these predictions neurons with s-OLs showed a reduced probability for action potential burst firing during $\left[\mathrm{K}^{+}\right]_{\mathrm{o}}$ elevations. These data suggest that $\mathrm{s}$-OLs are integrated into a glial syncytium for the millisecond rapid $\mathrm{K}^{+}$uptake limiting activity-dependent $\left[\mathrm{K}^{+}\right]_{0}$ increase in the perisomatic neuron domain.

\footnotetext{
${ }^{1}$ Axonal Signalling Group, Netherlands Institute for Neuroscience, Royal Academy of Arts and Sciences, Meibergdreef 47, 1105 BA Amsterdam, The Netherlands. ${ }^{2}$ Cell Biology, Department of Biology, Faculty of Science, University of Utrecht, $3584 \mathrm{CH}$ Utrecht, The Netherlands. Correspondence and requests for materials should be addressed to M.H.P.K. (email: m.kole@nin.knaw.nl).
} 
$\mathrm{N}$ euron-glia interactions are critical for diverse functions of the central nervous system. Recent work shows that activity of glia modulates long-term synaptic potentiation or depression ${ }^{1-3}$, controls rhythmic network activity ${ }^{4}$ and contributes to neuronal dysfunction ${ }^{5}$. A tight organization of the glial syncytium around neurons has previously been observed to contribute to regulation of neuronal activity ${ }^{6,7}$, suggesting essential functional roles of local glia arrangements. In the grey matter, all glia cell types, including astrocytes and microglia, can be found in a satellite position around neurons but oligodendrocytes (OLs) are most frequently observed ${ }^{8-10}$. These OLs are referred to as perineuronal or satellite OLs (s-OLs) ${ }^{9,11-13}$. The present view of $\mathrm{s}$-OLs is that they are primarily non-myelinating ${ }^{10,12,14,15}$ in contrast to the main function of OLs as myelin-producing cells ${ }^{16}$. However, s-OLs may provide metabolic support for neurons ${ }^{10}$, protect neurons against apoptosis ${ }^{17}$ or remyelinate axons following demyelinating injuries ${ }^{12}$. Since s-OLs are often located at the base of the soma ${ }^{13}$, close to the action potential (AP) initiation site at the axon initial segment (AIS) ${ }^{18}$, we here hypothesized that their privileged position enables s-OLs to influence AP firing.

To investigate the functional and anatomical properties of s-OLs, we combine simultaneous whole-cell patch-clamp recordings and live-confocal imaging with post hoc immunofluorescence and electron microscopy of neuron-s-OL pairs in acute neocortical slices from adult mice. We find that s-OLs myelinate surrounding axons and exhibit time-locked $\mathrm{Ba}^{2+}$ - and carbenoxolone-sensitive inward currents in response to APs generated in the host neurons. Unexpectedly, the AP-evoked inwardly rectifying $\mathrm{K}^{+}$(Kir) currents are not mediated by somatically expressed OL-specific Kir4.1 channels. Instead, the AP-evoked currents reflect junctional coupling to nearby astrocytes forming together with s-OLs a perisomatic glial syncytium. Moreover, during repetitive high-frequency AP firing of pyramidal neurons, s-OL coupled syncytia constrain the neuronal AP generation during periods of fast accumulation of $\left[\mathrm{K}^{+}\right]_{\mathrm{o}}$. These results suggest that $\mathrm{s}$-OLs within a glial syncytium perform multiple functional and anatomical roles, spatially buffering $\mathrm{K}^{+}$and myelinating axons within the perisomatic domain.

\section{Results}

Distribution of s-OLs around neocortical layer 5 neurons. To study s-OLs, we used a transgenic mouse line that expressed enhanced cyan fluorescent protein (ECFP) under control of a PLP promoter (PLP-ECFP, see Experimental Procedures; Fig. 1a) and focused on the neocortical layer 5 region. Electron microscopy identification of ECFP expressing s-OLs (Fig. 1b) revealed a characteristic very close apposition to neuronal cell membranes over long stretches with an interstitial space of $12.3 \pm 1.8 \mathrm{~nm}$ $(n=4$ cells). A close apposition was also seen in confocal analyses of neuron-s-OL pairs in which s-OLs were often found to fit in concavely shaped membrane regions of layer 5 neuron cell bodies and primary basal dendrites (Fig. 1c; Supplementary Movie 1). Estimates of the opposing membrane area yielded an average of $83 \pm 8 \mu \mathrm{m}^{2}$ (range: $60-110 \mu \mathrm{m}^{2}, n=6$ ). The initial description of $\mathrm{s}$-OLs noted that these cells often locate to the base of the neuron soma ${ }^{13}$. To examine whether s-OLs have a preferred location around the pyramidal neuron cell body, we analysed tissue labelled for OLs and neurons. Confocal $z$-stack images had on average $46 \pm 5$ OLs (24 regions of interest (ROIs), 3 animals and $n=1,103$ cells) with $34.4 \pm 1.4 \%$ being s-OLs $(n=380)$. From all NeuN-labelled neurons, $18.4 \pm 2.0 \%$ were associated with a s-OL (Supplementary Fig. 1a,b). However, this likely underestimates the percentage of s-OLs at larger pyramidal neurons, since s-OLs
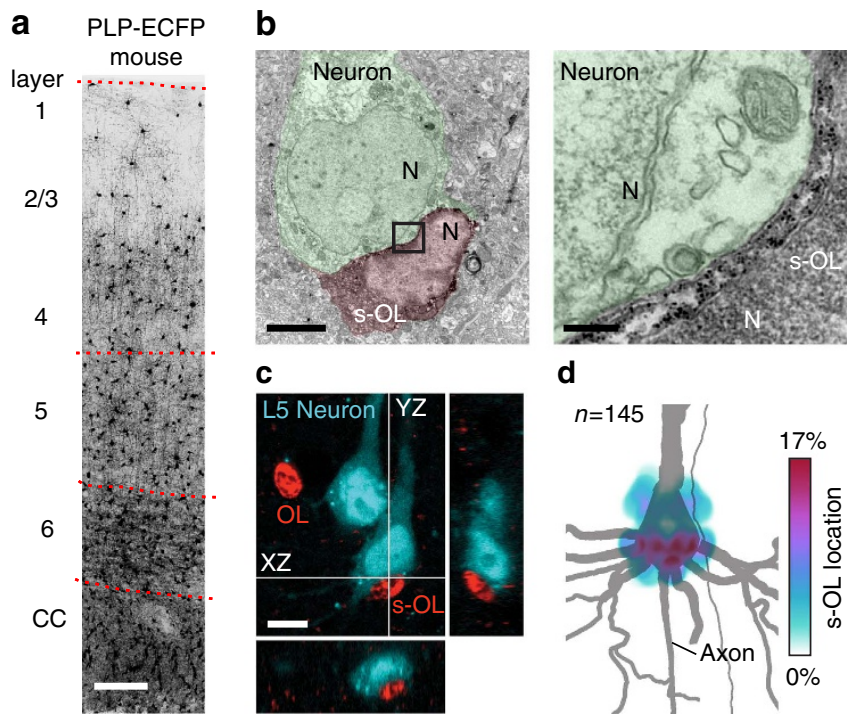

d

Figure 1 | Identification and distribution of s-OLs around pyramidal neurons. (a) Overview image of oligodendrocytes in the somatosensory neocortex labelled for ECFP in the transgenic PLP-ECFP mouse. Scale bar, $150 \mu \mathrm{m}$. (b) Left: s-OL (red pseudo-colour) in a PLP-ECFP mouse neocortex exhibits positive immuno-gold labelling in the cell body. The host neuron is pseudocoloured in light green and nuclei are labelled with N. Scale bar, $3 \mu \mathrm{m}$. Right: higher magnification image of the box indicated in the left image, which shows a distinct and confined location of gold particle-labelled ECFP in the cell body (black dots). Scale bar, $200 \mathrm{~nm}$. (c) Confocal image of labelled OLs in a PLP-ECFP mouse and two biocytin-filled L5 neurons and corresponding orthogonal views. The $\mathrm{s}-\mathrm{OL}$ cell body is in direct contact with the neuronal soma. Processes of OLs are not visible because laser intensity was reduced to a minimum. Scale bar, $10 \mu \mathrm{m}$. Also, see Supplementary Movie 1. (d) Heatmap illustrating the location probability of $145 \mathrm{~s}-\mathrm{OLs}$ in relation to the soma of an example L5 neuron (grey). s-OLs were positioned at multiple locations around the soma, but the highest percentages were found around the base of neurons close to the AIS and the basal dendrites. See also Supplementary Fig. 1.

preferentially reside near glutamatergic neurons ${ }^{10}$ and NeuN labels all neurons. For 145 randomly selected s-OLs, we plotted their position relative to the neuron soma. The data showed that s-OLs are positioned with higher probability around the soma base and basal dendrites (51.4\%) than in the vicinity of the apical dendrite $(21.5 \%)$ or between these subcellular locations $(27.1 \%$, $\chi^{2}$-test, $P<0.0001, n=3$ mice; Fig. 1d). Simultaneous labelling for OLs, $\beta$-IV-spectrin (an AIS marker) and NeuN revealed that the edge of s-OLs was on average $7.4 \pm 1.4 \mu \mathrm{m}$ away from the onset of the AIS ( $n=26$, Supplementary Fig. 1c).

Identified layer 5 s-OLs myelinate axons. For electrophysiological recording and single-cell fluorescence characterization, we targeted putative s-OLs based on their position at the soma of medium- to large-sized layer 5 pyramidal neurons (Fig. 2a,b). s-OLs were recorded either individually (total $n=70$ ) or during s-OL-neuron paired recordings (total $n=71$ pairs). While various glia cell types can be in contact with neurons, OLs are most frequently in a satellite position ${ }^{9}$. In line, satellite glia cells of wild-type (WT) animals were in some cases astrocytes or OL precursor cells (combined $\sim 20 \%$; Supplementary Fig. 1d). Quantification of the soma area from 16 neuron-s-OL pairs revealed that the soma size of s-OLs significantly and positively scaled with the host neuron cell body dimensions (Fig. 2c). Live-confocal scans showed that all s-OLs had internode-like 
a

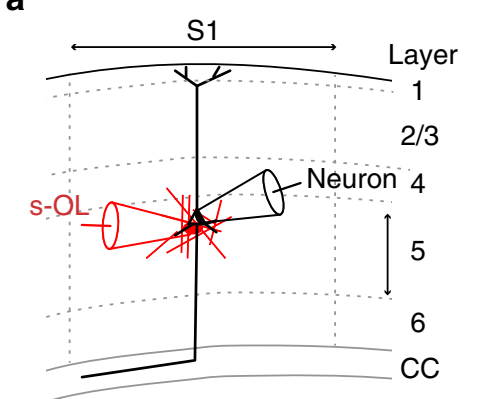

b
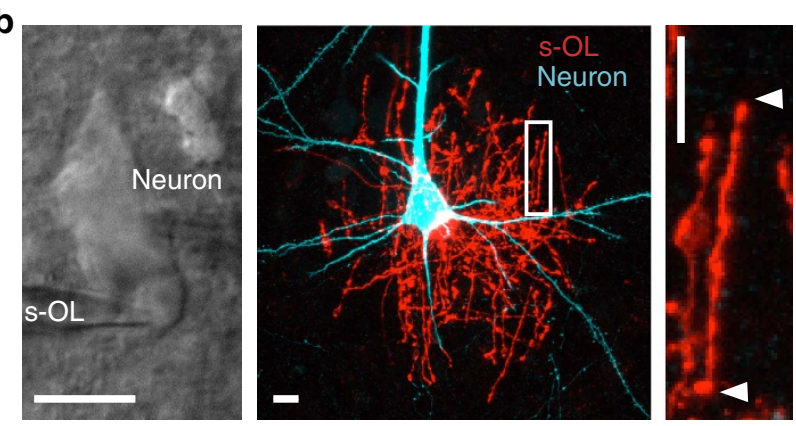

d

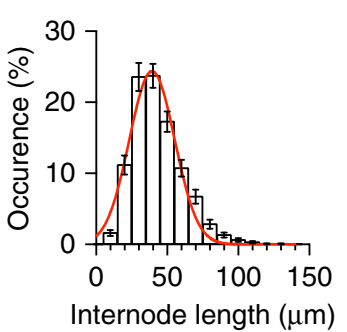

e

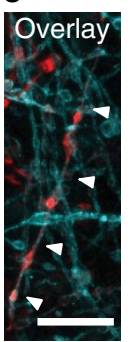

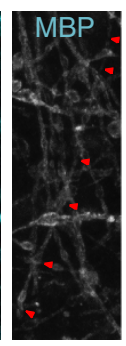

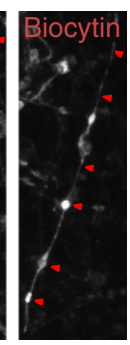

f

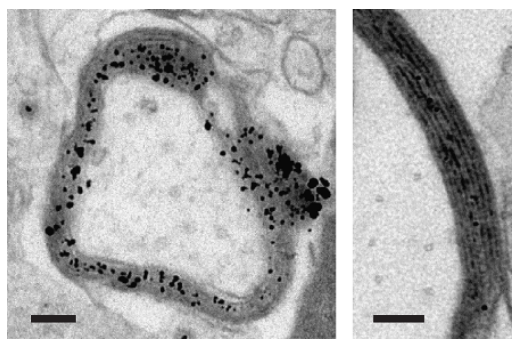

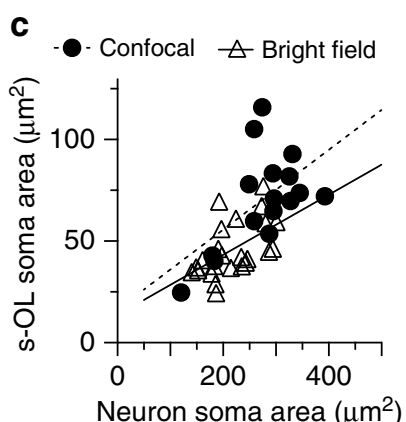

Neuron soma area $\left(\mu \mathrm{m}^{2}\right)$

g

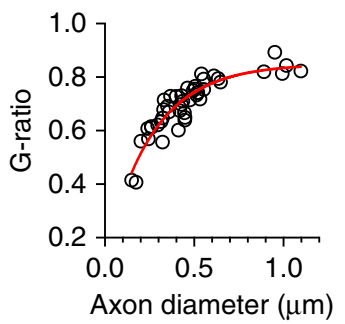

Figure 2 | Experimental targeting of s-OLs reveals compact myelin and wrapping of surrounding axons. (a) Illustration of the experimental approach. $\mathrm{s}-\mathrm{OL}$ (red) and layer 5 neuron (black) targeted with patch-clamp pipettes in mouse primary somatosensory neocortex. (b) Left: Bright-field image of a neurons-OL pair. Patch pipettes are visible. Middle, right: Confocal maximum z-projected image of a neuron-s-OL pair after intracellular loading with Alexa 488 (neuron) and Alexa 594 (s-OL) dyes. The white box is displayed at higher magnification on the right. White arrows indicate one internode. All scale bars, $10 \mu \mathrm{m}$. See Supplementary Movie 2. (c) Correlation of soma size between neurons and s-OLs as estimated from two separate data sets. Closed circles display data from maximum $z$-projected live-confocal scans and open triangles are measurements from bright-field images. Both data sets were fit by linear regressions (confocal scans: $n=16$, correlation coefficient $r^{2}=0.33, P=0.02$; bright field: $n=24, r^{2}=0.29, P=0.007$ ). (d) Distribution histogram of s-OL internode length measured from confocal live image $z$-stacks $\left(n=32\right.$ cells). The data were fit by a Gaussian function (red line, $y=24.36^{\star} e\left(-0.5^{\star}((x-39.30) /\right.$ $\left.15.93)^{2}\right)$ ). Data presented are mean \pm s.e.m. (e) A s-OL was filled with biocytin and Alexa 594 during recordings and subsequently post hoc labelled for myelin basic protein (MBP). Internodal structures are positively co-labelled by MBP and biocytin (red and white arrowheads). Scale bar, $10 \mu \mathrm{m}$. (f) Left: electron microscopy image of an identified horseradish peroxidase (HRP)-filled transversal cut s-OL process shows multiple layers of myelin wrapped around an axon. Gold particles are visible as black dots in the outer tongue and all myelin layers, suggesting a direct cytoplasmic connection with the cell body. Right: a putatively different axon at higher magnification shows about seven gold-labelled compact myelin wraps. See also Supplementary Fig. 2 . Scale bars, $100 \mathrm{~nm}$. $(\mathbf{g})$ The g-ratio measured from single identified s-OL axons $(n=45)$ plotted as function of the axon diameter. s-OLs myelinate axons of variable diameter. The data were fitted with an exponential equation $(y=-0.723 \times \mathrm{e}(-0.0038 \times x)+0.85)$.

structures oriented in vertical, horizontal and various diagonal directions, and resembled myelin based on the transmitted light images (Fig. 2b; Supplementary Movie 2; Supplementary Fig. 2a). s-OLs had on average $32 \pm 2$ internodal-like branches with a mean length of $43.4 \pm 1.3 \mu \mathrm{m}(n=32$; Fig. $2 \mathrm{~d})$.

Myelination by s-OLs is unexpected as s-OLs are referred to as non-myelinating in the literature ${ }^{10,12,14,15}$. To further examine whether axon wrapping by s-OLs forms compact myelin, we pursued two independent approaches. First, biocytin- and Alexa 594-filled s-OLs were positively post hoc labelled for myelin basic protein (MBP; $n=4$; Fig. 2e). Second, to identify single s-OL internode-axon assemblies at the ultrastructural level, we filled s-OLs ( $n=5$ cells) with horseradish peroxidase. Electron microscopy processing (Supplementary Fig. 2b) revealed that s-OLs made on average $\sim 4$ myelin wraps (range: $3-7, n=12$; Fig. 2f) around axons with diameters ranging between 0.2 and $1.1 \mu \mathrm{m}$ (Fig. 2g; Supplementary Fig. 2c). The average g-ratio was $0.70 \pm 0.02$ ( $n=45$ axons, $n=5$ s-OLs; Fig. $2 \mathrm{~g}$ ). Notably, we found that s-OLs never myelinated the first internode of their host neuron. This is most likely explained by the length of the first internode of the layer 5 axon extending up to $\sim 110 \mu \mathrm{m}$ from the soma edge, which is beyond the maximum expansion of the $\mathrm{s}$-OL internodes $(\sim 80 \mu \mathrm{m}$ from the centre of the s-OL cell body, $n=40$ ). These data highlight that all s-OLs myelinate axons of varying small diameter with short internodal processes, classifying them as type $1 \mathrm{OLs}^{15}$.
s-OLs sense APs at high temporal resolution. Whole-cell recordings revealed that $s$-OLs have a resting membrane potential of on average $-86 \mathrm{mV}$, a very low input resistance $\left(R_{\mathrm{N}}\right)$ of $\sim 13 \mathrm{M} \Omega$ and a linear current-voltage relationship (Supplementary Table 1; Supplementary Fig. 3a-c). Given the close proximity to layer 5 somata and AIS, we tested in dualwhole-cell recordings (Fig. 3a) whether s-OLs respond to APs. In response to a single AP, s-OLs displayed a small and transient outward current $(12.5 \pm 0.3 \mathrm{pA}, n=23)$ temporally aligned with the capacitive charge during the AP depolarization (Fig. 3b). The capacitive transient was followed by an inward current with amplitude of on average $-2.2 \pm 0.3 \mathrm{pA}$ ( $n=7$; Fig. $3 c$ ). Interestingly, the onset of this inward current aligned with the beginning of the repolarization of the AP and continued for several hundreds of milliseconds (Fig. 3d). Subthreshold current injections in neurons did not lead to detectable inward currents $(n=6$; Supplementary Fig. 3d). Since layer 5 neurons can generate APs at frequencies up to $\sim 300 \mathrm{~Hz}$ (ref. 19), we assessed the impact of a high-frequency train of APs and the response of s-OLs (Fig. 3e). Five APs with a frequency of $100 \mathrm{~Hz}$ resulted in an inward current of $-15.9 \pm 1.8 \mathrm{pA}$ in amplitude, cumulating with each AP and decaying double exponentially $\left(\tau_{1}=39 \pm 4 \mathrm{~ms}\right.$, $\tau_{2}=235 \pm 24 \mathrm{~ms}, \tau_{\text {weighted }}=74 \pm 9 \mathrm{~ms}, n=22$ ). Due to the low input resistance in current-clamp configuration, five APs depolarized s-OLs by only $0.5 \pm 0.1 \mathrm{mV}(n=5$; Fig. $3 e)$. The charge transferred per AP remained constant between 1 and 
a

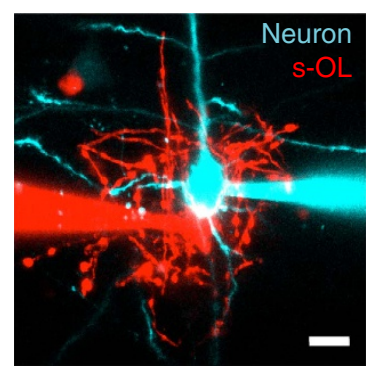

b

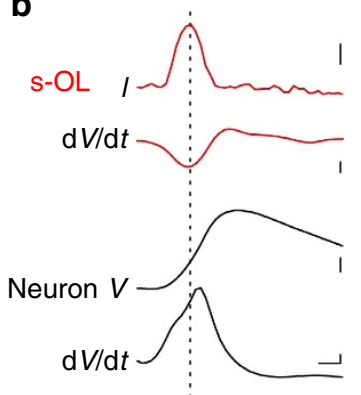

c

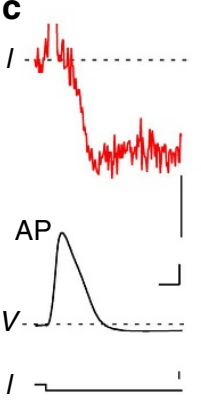

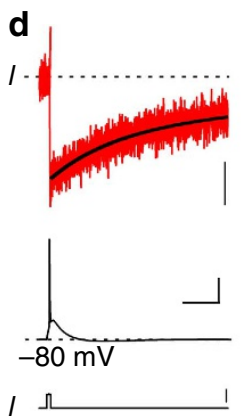

e

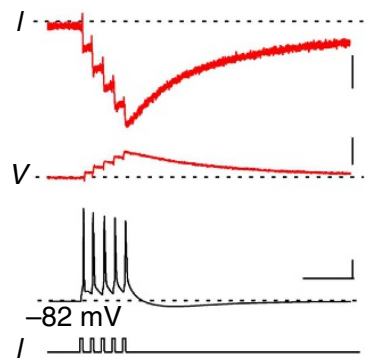

f

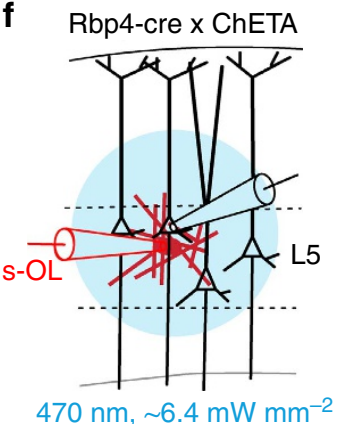

g
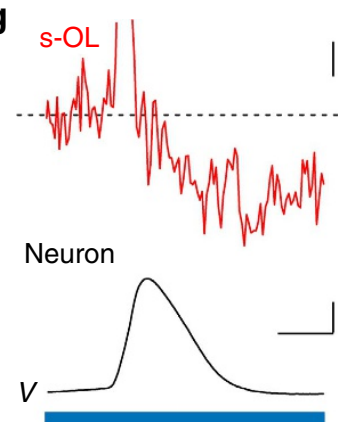

h
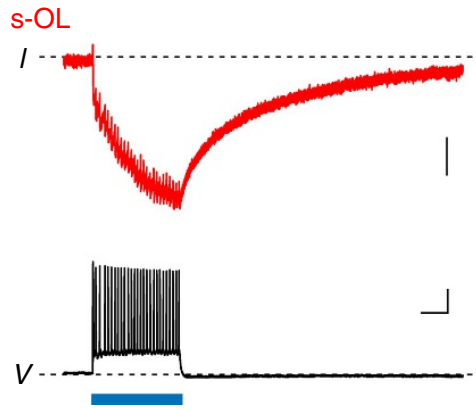

Figure 3 | Action potentials evoke time-locked inward currents in s-OLs. (a) Confocal z-projected image of dual-whole-cell recording from L5 neuron-s-OL pair in the somatosensory neocortex. Scale bar, $20 \mu \mathrm{m}$. (b) The $\mathrm{dV} / \mathrm{d} t$ of the AP aligns with the fast current transient in the s-OL, reflecting the capacitive charge of the s-OL membrane during the rising phase of the AP. In current clamp, the s-OL membrane voltage change is slow (dV/dt) compared with the neuronal AP. Note the $\mathrm{dV} / \mathrm{d} t$ is from a different recording and filtered for display. The other traces are from the same cell as in $\mathbf{c}$ and $\mathbf{d}$. Scale bars, (top to bottom) $10 \mathrm{pA}, 1 \mathrm{Vs}^{-1}, 30 \mathrm{mV}$ (middle), $100 \mathrm{Vs}^{-1}$ and $0.1 \mathrm{~ms}$. (c) AP repolarization temporally aligns with a rapid inward current in the s-OL. Average of 35 trials; scale bars, $5 \mathrm{pA}, 30 \mathrm{mV}, 500 \mu \mathrm{s}$ and $0.7 \mathrm{nA}$. Capacitive current transient clipped for clarity. (d) The single AP evoked a rapid inward current in the s-OL (red trace, top) that decayed slowly (held at $-91 \mathrm{mV}$; black fit, $\tau_{1}=78 \mathrm{~ms}, \tau_{2}=451 \mathrm{~ms}$ ). Traces displayed are the average of 25 trials. Scale bars, $2 \mathrm{pA}, 30 \mathrm{mV}, 50 \mathrm{~ms}$ and $1 \mathrm{nA}$. (e) Five APs evoked at $100 \mathrm{~Hz}$ potentiated the inward current amplitude in the s-OL (clamped to $-88 \mathrm{mV}$, upper red trace). In current clamp (lower trace), the s-OL was depolarized by $\sim 500 \mu \mathrm{V}$. Traces are average of 26 trials. Scale bars, $10 \mathrm{pA}, 500 \mu \mathrm{V}, 30 \mathrm{mV}$ and $50 \mathrm{~ms}$, respectively. (f) Illustration of the targeted activation of layer 5 neurons by ChR2 while simultaneously recording from s-OL-neuron pairs. (g) Single light-evoked neuronal AP led to a capacitive transient (clipped) in the s-OL, followed by an inward current that activated during AP repolarization similar to electrical evoked AP. Scale bars, $2 \mathrm{pA}, 30 \mathrm{mV}$ and $0.5 \mathrm{~ms}$. (h) The synchronised network activation of layer 5 neurons by ChR2 led to a large AP evoked inward current in a simultaneous recorded single s-OL. Scale bars, $20 \mathrm{pA}$ (top), and $30 \mathrm{mV}$ and $0.2 \mathrm{~s}$ (bottom).

$200 \mathrm{~Hz}$ (Kruskal-Wallis test, $P=0.5, n=35$; Supplementary Fig. $3 \mathrm{e}$ ) and was on average $0.42 \pm 0.05 \mathrm{pC}$ per AP. Varying the holding potential of s-OLs to positive voltages diminished, but did not abolish, the inward currents (Supplementary Fig. 3f). We next assessed the impact of a synchronously activated network of layer 5 neurons by photostimulating layer 5 neurons that expressed channelrhodopsin 2 (ChR2, ref. 20; Fig. 3f). Single APs of the host neuron were well discernible and similar to the response of electrically evoked inward currents (Fig. 3g). During long light pulses, inward currents had an average amplitude of $-92 \pm 19 \mathrm{pA}$ with a total charge of $-136 \pm 36 \mathrm{pC}$ that decayed double exponentially ( $\tau_{\text {weighted }}=377 \pm 113 \mathrm{~ms}, n=6$; Fig. $3 \mathrm{~h}$ ). These results show that during in vivo-like synchronized activity of layer 5 pyramidal neurons, a substantial inward current is generated in s-OLs.

AP-evoked currents are glial specific and $\mathbf{B a}^{2+}$ sensitive. We next asked whether these inward currents are specific for s-OLs. Paired recordings of pyramidal neurons and astrocytes in a satellite position on average $2 \pm 1 \mu \mathrm{m}$ away $(n=5$, Mann-Whitney test, $P=0.18$, compared to s-OLs $(n=22))$ revealed inward currents of comparable amplitude $(-23.26 \pm 7 \mathrm{pA}, n=5$, Mann-Whitney test, $P=0.4)$ and decay kinetics $\left(\tau_{\text {weighted }}=64 \pm 9 \mathrm{~ms}, n=5\right.$, MannWhitney, $P=0.62$, Fig. 4a,b). In contrast, AP-evoked inward currents were absent in interneurons that were in a satellite position to pyramidal neurons $(-0.94 \pm 0.3 \mathrm{pA}, n=4$, Fig. $4 \mathrm{a}, \mathrm{b})$.
OLs that were not in a satellite position to the recorded neuron ( $>10 \mu \mathrm{m}$ membrane separation) had significantly smaller inward currents in response to APs $(-2.1 \pm 1.1 \mathrm{pA}, n=8$, Mann-Whitney, $P=0.0002$, Fig. 4b) and were not detectable beyond an intercellular distance of $50 \mu \mathrm{m}(n=4)$.

To identify the source of the inward current, we first applied blockers against ionotropic glutamate receptors, known to be expressed in adult OLs and OPCs ${ }^{21,22}$. However, the AP-evoked inward currents were unaffected by the NMDA ( $N$-methyl-Daspartate) receptor blocker D-AP5 $(50 \mu \mathrm{M})$ or $20 \mu \mathrm{M}$ of the kainate/AMPA ( $\alpha$-amino-3-hydroxy-5-methyl-4-isoxazole propionic acid) receptor blocker CNQX (ratio (ctrl/blocker) of $1.1 \pm 0.1$ and $0.9 \pm 0.1$, respectively, Wilcoxon signed-rank test, $P=0.99, n=3 ; P=0.65, n=2$; Fig. 4c). Similarly, blockers against $\mathrm{GABA}_{\mathrm{A}}(\gamma$-aminobutyric acid; $5 \mu \mathrm{M}$ gabazine $)$ and $\mathrm{GABA}_{\mathrm{B}}(50 \mu \mathrm{M}$ CGP-35348) receptors also did not affect the inward currents (0.96- and 1.04-fold change, respectively, Wilcoxon signed-rank test, $P=0.65, n=3 ; P=0.18, n=2$; Fig. $4 c$ ). Adult rat OLs express inwardly rectifying potassium $\left(\mathrm{K}^{+}\right)$channels at the cell body that are sensitive to barium $\left(\mathrm{Ba}^{2+}\right)^{23}$. Following $100 \mu \mathrm{M} \mathrm{Ba} \mathrm{Ba}^{2+}$ application, the maximum amplitude of the AP-evoked inward current was reduced by $80.7 \pm 2.6 \%$ (Wilcoxon signed-rank test, $P=0.043, n=5$; Fig. 4c) and the decay time constant of the remaining current was significantly slowed (control: $\tau_{\text {weighted }}=58 \pm 8 \mathrm{~ms}, \quad \mathrm{Ba}^{2+}: \quad \tau_{\text {weighted }}=505 \pm 148 \mathrm{~ms}, \quad n=5$, Wilcoxon signed-rank test, $P=0.04$ ). 
a

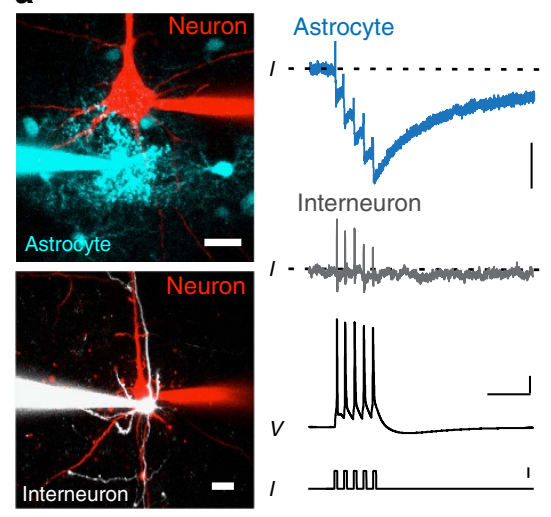

b

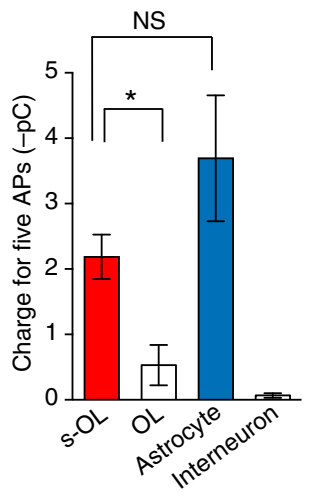

C
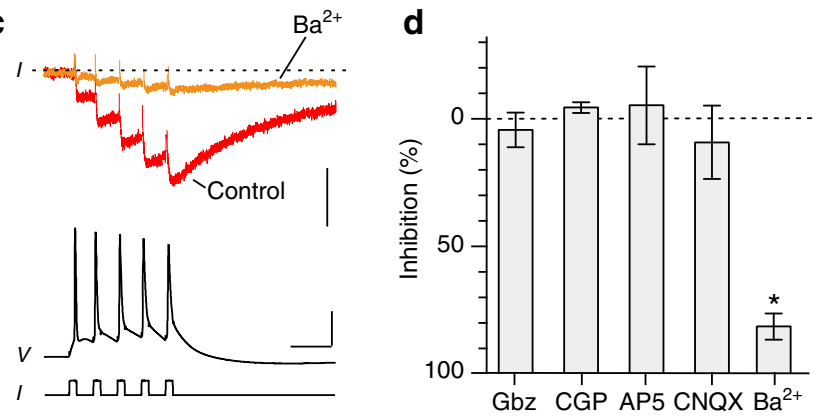

Figure 4 | Inward currents are glial specific and $\mathbf{B a}^{2}+$ sensitive.

(a) Left: Z-projected confocal images of paired neuron-astrocyte and neuron-interneuron recordings. Scale bars, $20 \mu \mathrm{m}$. Right: traces from paired recordings show that astrocytes in satellite position show similar inward currents as s-OLs, but interneurons exhibit no inward currents; capacitive transients are still visible. The electrophysiology traces do not correspond to the image shown on the left. Scale bars, $10 \mathrm{pA}$ (top and middle) and $30 \mathrm{mV}$, and $50 \mathrm{~ms}$ and $1 \mathrm{nA}$ (bottom). (b) Summary data of the charge for five APs for s-OLs $(n=22)$, oligodendrocytes not in satellite position $(n=8)$, astrocytes $(n=5)$ and interneurons $(n=4)$ reveal that only glia in close proximity to the firing neuron exhibit a substantial inward current. Mann-Whitney test, $P=0.0004$ and $P=0.129$. Data are mean \pm s.e.m. (c) Left: extracellular application of $100 \mu \mathrm{M} \mathrm{Ba}^{2+}$ (orange) blocks the AP-induced inward current in s-OLs. Scale bars, 20 pA, $40 \mathrm{mV}$ and $20 \mathrm{~ms}$. (d) Population data of blocking experiments. Neither glutamatergic $(50 \mu \mathrm{M}$ D-AP5, $n=3 ; 20 \mu \mathrm{M}$ CNQX, $n=2)$ nor GABAergic receptor blockers ( $5 \mu \mathrm{M}$ gabazine, $n=3 ; 50 \mu \mathrm{M}$ CGP-35348, $n=2$ ) affected the inward current (for all Wilcoxon signed-rank test, $P>0.18$ ). In contrast, $100 \mu \mathrm{M} \mathrm{Ba}^{2+}$ led to an $\sim 80 \%$ reduction of the $\mathrm{s}-\mathrm{OL}$ inward current (Wilcoxon signed-rank test, $n=5, P=0.043$ ). Data shown are mean \pm s.e.m.

On the basis of previous recordings of voltage-gated $\mathrm{K}^{+}$ currents in somatic outside-out patches from rat L 5 neuron $\mathrm{s}^{24}$ the $\mathrm{K}^{+}$charge per AP was estimated to be $5.3 \mathrm{fC} \mu \mathrm{m}^{-2}$. The L5 soma width (14.4 $\pm 0.3 \mu \mathrm{m}, n=34)$ accounted for a somatic surface area of $\sim 707 \mu \mathrm{m}^{2}$ (assuming a ball) and a single AP would thus generate a $\mathrm{K}^{+}$charge of $3.7 \mathrm{pC}$. An individual $\mathrm{s}$-OL, generating $0.42 \mathrm{pC}$ charge per AP, thereby takes up $\sim 11 \%$ of the somatically extruded $\mathrm{K}^{+}$ions. Assuming a cleft dimension $(\delta)$ of $20 \mathrm{~nm}$ (or $0.02 \mu \mathrm{m}$ ) between the neuron and the s-OL (Fig. 1b; Supplementary Movie 1), the numerical estimation of the $\mathrm{K}^{+}$charge within the extracellular volume would be equivalent to $2.7 \mathrm{mM}$ per AP (see Methods). Taken together, these data show that $\mathrm{Ba}^{2+}$-sensitive inward currents are specific for glia cells in direct contact or close proximity to the firing neuron, suggesting that they play a role in the activity-dependent $\mathrm{K}^{+}$uptake.
Kir4.1 channels are not mediating inward $\mathrm{K}^{+}$currents. On the basis of our experiments (Figs 3 and 4) and a report of $\mathrm{Ba}^{2+}$. sensitive inward currents in OPCs and adult $\mathrm{OLs}^{23,25}$, we hypothesized that the potassium channel Kir4.1 is responsible for the observed inward current after AP firing. To test this hypothesis, we utilized a floxed Kir4.1 mouse ${ }^{26}$ in which we specifically removed Kir4.1 in adult OLs. After conditionally knocking out Kir4.1 (Kir4.1 ${ }^{-/}$) the deletion was confirmed by the absence of immuno-gold labelling at electron-dense OL cell bodies (Fig. 5a,b; Supplementary Fig. 4) ${ }^{9}$. In WT, gold particles were found in cell bodies but never in myelin sheaths, which is in agreement with previous published data ${ }^{27,28}$. To examine the consequence of Kir4.1 loss in OLs, we assessed the $\mathrm{Ba}^{2+}$-sensitive current in voltage clamp of Kir4.1-1- and WT s-OLs (Fig. 5c). The $\mathrm{Ba}^{2+}$-sensitive conductance as estimated from steady-state $\mathrm{I}-\mathrm{V}$ curves was significantly smaller in Kir4.1 $1^{-1-}$ mice $(4.1 \pm 2 \mathrm{nS}, \quad n=5)$ compared with WT s-OLs $(13.1 \pm 1.9 \mathrm{nS}, n=6$, Mann-Whitney test, $P=0.017$; Fig. 5c,d; Supplementary Fig. 5a-c). Moreover, consistent with the sensitivity of Kir4.1 to intracellular spermine ${ }^{29}$, the $\mathrm{Ba}^{2+}$-sensitive current of wild-type s-OLs was modulated and reduced in outward rectification when spermine was added intracellularly (Supplementary Fig. 5d).

Next, we assessed in paired recordings from neurons and s-OLs the consequence of Kir4.1 knockout on $\mathrm{K}^{+}$inward currents evoked by APs (Fig. 5e,f). Unexpectedly, an inward current was recorded in all pairs without a difference in their maximum amplitude (unpaired t-test, $P=0.81$ ), charge (Mann-Whitney test, $P=0.6$ ) or the weighted decay time constant (unpaired $t$-test, $P=0.09$ ) compared with WT (WT: $n=16$; Kir4.1 $1^{-1-}$ : $n=9$; Fig. 5g, WT data set same as Fig. 3). To further analyse the origin of the AP-mediated current in Kir4.1 $1^{-/-} \mathrm{s}$-OLs, we applied $\mathrm{Ba}^{2+}$. Similar to WT s-OLs $100 \mu \mathrm{M} \mathrm{Ba}^{2+}$ reduced the AP-evoked current in Kir4.1 $1^{-I-}$ s-OLs by $86 \pm 1 \%(n=4$ Mann Whitney test, $P=0.11$ ). In line with these data, $s$-OLs from Kir4.1 ${ }^{-1-}$ animals appeared indistinguishable in their resting membrane properties compared with WT s-OLs (Supplementary Table 1). Thus, despite successful deletion of Kir4.1 in OLs and a reduction of $\mathrm{Ba}^{2+}$-sensitive current, the AP-dependent inward current and resting membrane properties are maintained. These data either suggest that other members of the Kir family or gap-junction coupled astrocytes contribute to the observed inward current.

s-OLs connect to the astrocytic syncytium. Panglial dye coupling has been reported in acute grey and white matter slices ${ }^{30-32}$, electrical coupling between OLs and astrocytes has been shown to occur in explant cultures ${ }^{33}$ and gap-junctions are implicated in $\mathrm{K}^{+}$buffering ${ }^{34}$. We therefore tested reciprocal electrical gap-junction coupling between the pairs of s-OLs and between s-OLs and neighbouring astrocytes (Fig. 6a,b). The average coupling ratio between s-OLs and astrocytes $(n=12)$ was $3 \pm 1 \%$ and between OL-OL pairs $(n=3) 0.6 \pm 0.1 \%$ (Mann-Whitney test, $P=0.0044$; Fig. $6 c$ ) when recorded from similar distances (Mann-Whitney test, $P=0.82$ ). Interestingly, the coupling was distance dependent with a strong attenuation at distances $>20 \mu \mathrm{m}$ (Fig. 6c). In addition, astrocytes were dye coupled with $9 \pm 1$ other astrocytes within a domain $(n=20)$. To test whether electrical coupling between s-OLs and astrocytes is mediated by gap-junctions, we bath applied $100 \mu \mathrm{M}$ carbenoxolone (CBX). After wash in the voltage coupling between s-OLs and astrocytes was reduced by $67 \pm 6 \%$ (Wilcoxon signed-rank test, $P=0.03, n=6$; Fig. 6d). Finally, we tested the impact of CBX on the AP-evoked inward current in s-OLs. CBX caused a reduction in s-OL current amplitude by $58.1 \pm 4.4 \%$ (Wilcoxon signed-rank test, $P=0.043 n=5$; Fig. 6e) 
a

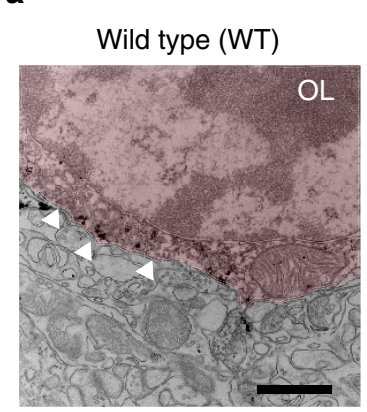

b

PLP-Cre $\times$ Kir4. $1^{\mathrm{fl} / \mathrm{fl}-/-}$

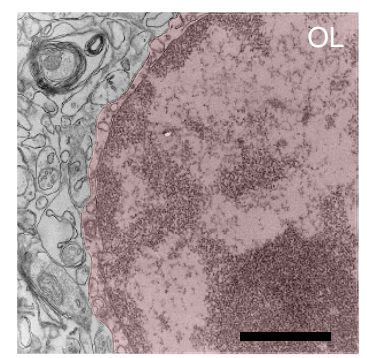

c

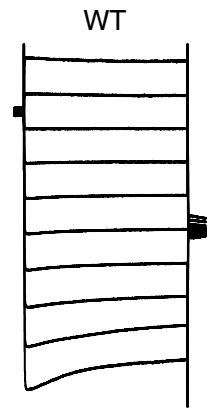

Kir4.1 $1^{-/-}$

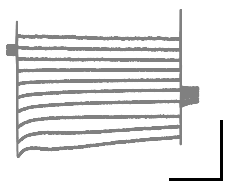

$14 \mathrm{mV}$

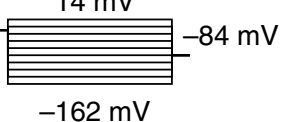

$-162 \mathrm{mV}$ d $\circ \mathrm{WT}(n=6)$ Kir4.1 $1^{-/-}(n=5)$

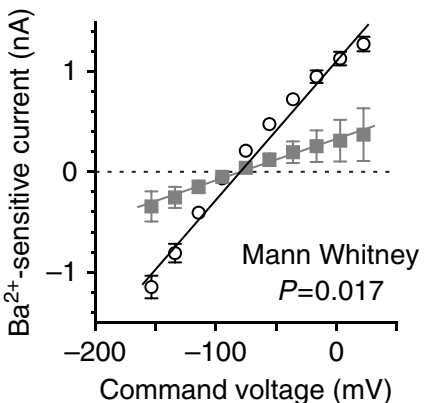

e

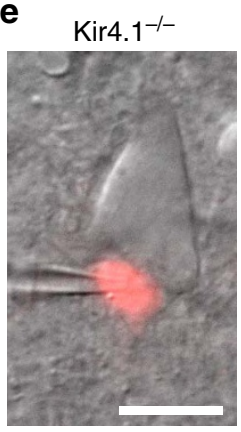

f

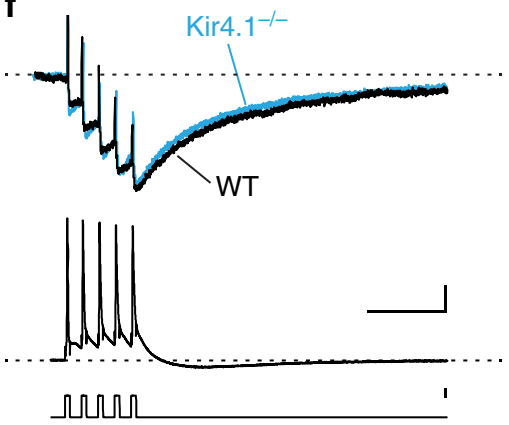

g

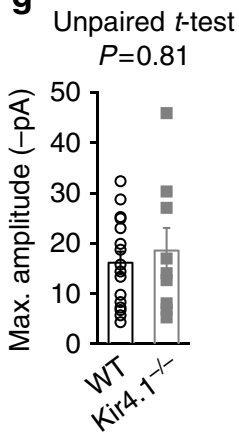

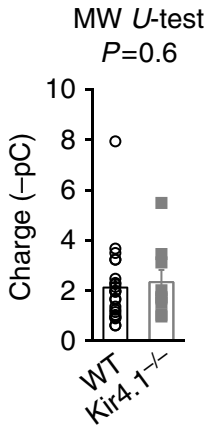

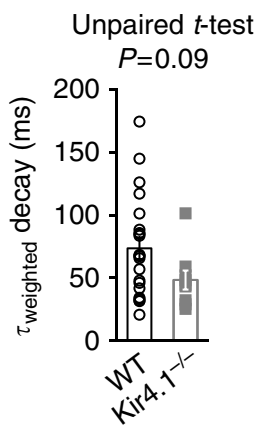

Figure 5 | Oligodendrocytic Kir4.1 does not contribute to AP-evoked inward currents in s-OLs. (a,b) Electron microscopy images of WT and Kir4.1-/ tissue immuno-gold labelled with an antibody against Kir4.1. In the WT tissue, gold particles are present (white arrowheads) at the soma that were absent in the Kir4.1-/- OLs. Scale bars, $1 \mu \mathrm{m}$. (c) Ba ${ }^{2+}$-sensitive currents from WT (black) and Kir4.1-/ - (grey) s-OLs evoked by the displayed voltage steps. Scale bars, $0.5 \mathrm{nA}$ and $0.1 \mathrm{~s}$. (d) Summary of the experiments in c shown in an $1-V$ curve. Kir4.1- / ${ }^{-} \mathrm{s}^{-O} \mathrm{OLs}$ have a reduced Ba ${ }^{+}-$-sensitive conductance. Data are mean \pm s.e.m. (e) Combined bright-field and tdTomato fluorescence example image of a Kir $4.1^{-/-} \mathrm{s}-\mathrm{OL}-\mathrm{neuron}$ pair in layer 5 . Scale bar, $10 \mu \mathrm{m}$. (f) Recording of the Kir4.1-/ $-\mathrm{s}-\mathrm{OL}-$ neuron pair shown in e overlaid with a recording from a WT experiment and the inward current evoked by five APs. $\mathrm{s}-\mathrm{OL}$ currents were normalized to the maximum amplitude. Scale bars, $30 \mathrm{mV}, 50 \mathrm{~ms}$ and $1 \mathrm{nA}$. (g) Summary data of the maximum amplitude, charge and weighted decay time constant reveal no differences of the action potential-evoked inward current properties between wild-type and Kir4.1- ${ }^{-}$s-OLs.

Data are mean \pm s.e.m.

without changing decay kinetics (control: $\tau_{\text {weighted }}=76 \pm 18 \mathrm{~ms}$, CBX: $\tau_{\text {weighted }}=136 \pm 46 \mathrm{~ms}, n=5$; Wilcoxon signed-rank test, $P=0.14)$. These results show that astrocytes and s-OLs in the vicinity of neuronal cell bodies are coupled by gap-junctions likely forming a syncytium to efficiently taking up $\mathrm{K}^{+}$from the extracellular perisomatic region.

Experimental and modelling predictions of potassium uptake. To examine how changes in $\left[\mathrm{K}^{+}\right]_{\mathrm{o}}$ relate to the s-OL inward currents, we applied $\mathrm{K}^{+}(1-30 \mathrm{mM})$ to the soma of s-OLs with pipettes closely positioned near the cell body $(7.2 \pm 0.4 \mu \mathrm{m}$, $n=21$; Fig. $7 \mathrm{a})$ and a holding potential of $-84 \mathrm{mV}$. A $\left[\mathrm{K}^{+}\right]_{\mathrm{o}}$ application of $1 \mathrm{mM}$ led to small transient outward currents (peak amplitude of $48.6 \pm 13.7 \mathrm{pA}, n=5$ ). However, when $\left[\mathrm{K}^{+}\right]_{\mathrm{o}}$ was higher than the control $\left[\mathrm{K}^{+}\right]_{\mathrm{o}}$ of $3 \mathrm{mM}$, inward currents increased up to a peak amplitude of on average $-514 \pm 106 \mathrm{pA}$ $(n=9$; Fig. $7 \mathrm{~b})$ at $30 \mathrm{mM}\left[\mathrm{K}^{+}\right]_{\mathrm{o}}$. By fitting these data with a linear regression $\left(R^{2}=0.99\right)$, we determined that the $\left[\mathrm{K}^{+}\right]_{\mathrm{o}}$ evoked peak currents reversed polarity at $3.2 \mathrm{mM}$. On the basis of the total s-OL surface area of $\sim 400 \mu \mathrm{m}^{2}$ and an apposition area of $\sim 80 \mu \mathrm{m}^{2}$ to the host neuron, we assumed that five times smaller current is generated at the s-OL soma during APs. In the adjusted linear regression $(y=-3.8 x+12.3)$, we substituted our peak amplitude current from five APs. Using the experimentally recorded range from -5 to $-30 \mathrm{pA}$ (Fig. $3 \mathrm{e}$ ), we calculated a range of concentrations from 4.51 to $11.0 \mathrm{mM}\left[\mathrm{K}^{+}\right]_{\mathrm{o}}$. The average increase was up to $7.2 \mathrm{mM}\left[\mathrm{K}^{+}\right]_{\mathrm{o}}$ for five APs equivalent to $\sim 1 \mathrm{mM}\left[\mathrm{K}^{+}\right]_{\mathrm{o}}$ rise per single AP. To evaluate the contribution of astrocytes, we applied CBX while puffing $30 \mathrm{mM}\left[\mathrm{K}^{+}\right]_{\mathrm{o}}$. The inward current reduced amplitude and charge by $52 \pm 8 \%(n=2$; Fig. 7c) indicating that gap-junction coupling to astrocytes may contribute for $\sim 50 \%$ of the inward current. These data suggest that the observed inward current is reflecting a participation of s-OLs in buffering $\left[\mathrm{K}^{+}\right]_{\mathrm{o}}$.

Next, we simulated the spatial $\mathrm{K}^{+}$charge profile by a single AP in a published spatially accurate computational model of a L5 pyramidal neuron (Supplementary Fig. 6a). The model revealed that the AIS, soma and proximal dendrites are the main membrane sites contributing to the $\mathrm{K}^{+}$efflux during the initiation and back propagation of a single AP strongly overlapping with the spatial arrangement of s-OLs. Given the critical role of the nearby AIS in determining the AP threshold in the axosomatic region ${ }^{35}$, we aimed to test whether local $\mathrm{K}^{+}$ buffering by the perisomatic syncytium influences neuronal excitability. Since $\mathrm{Ba}^{2+}$ is not specific for glial Kir, this excludes its use to distinguish the impact of glial and neuronal Kir. As an alternative, we first explored the impact of glial Kir-mediated $\mathrm{K}^{+}$buffering on neuronal excitability using a published computational model with simplified neuronal morphology ${ }^{36,37}$. Varying the intercellular distance between neuron and glia in this model between 10 and 2,000 $\mathrm{nm}$ showed that AP-evoked $\left[\mathrm{K}^{+}\right]_{\mathrm{o}}$ increased between 8 and $0.1 \mathrm{mM}$, respectively (Fig. 7d). As expected, the AP-evoked $\left[\mathrm{K}^{+}\right]_{\mathrm{o}}$ increase was highly dependent on the extracellular volume; logarithmically reducing the intercellular distance between the neuron and glia $(\delta)$ increased 
a

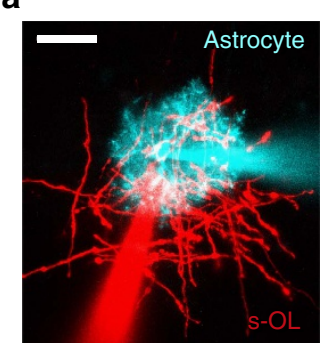

b

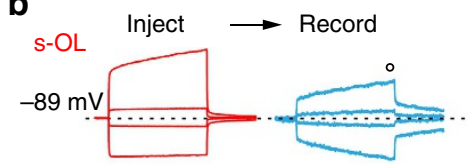

Astrocyte

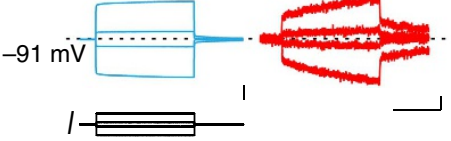

C. $\theta \cdot s-\mathrm{OL} / \mathrm{A} \neq \mathrm{s}-\mathrm{OL} / \mathrm{s}-\mathrm{OL}$
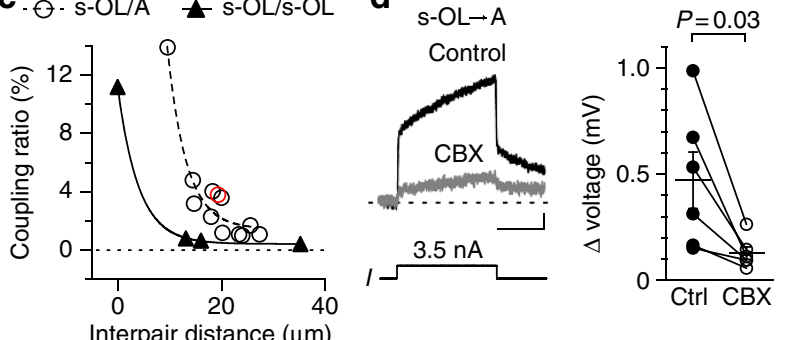

e $\mathrm{s}-\mathrm{OL}$
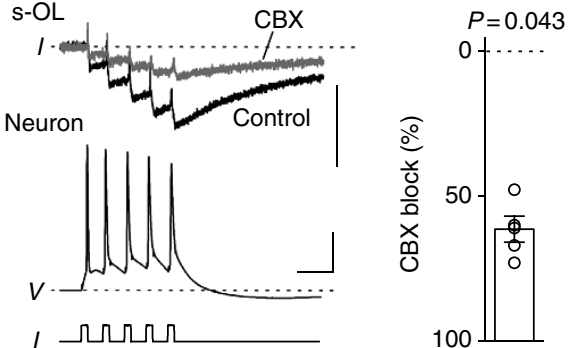

Figure 6 | s-OLs are gap-junction coupled to other s-OLs and astrocytes. (a) Confocal z-projected image of a s-OL and astrocyte. Scale bar, $30 \mu \mathrm{m}$. (b) Gap-junction coupling between s-OL and astrocytes was estimated by bidirectional current injections into s-OLs or adjacent astrocytes and simultaneous recordings of the response in the corresponding cell. Traces are averages of 10 trials from pair in $\mathbf{a}$. Open circle indicate the measurement region at the end of the pulse. Scale bars, (inject) $5 \mathrm{mV}$, (record) $200 \mu \mathrm{V}$ and $50 \mathrm{~ms}$, respectively. (c) Summary plot of the coupling ratio in the steady state as a function of the distance between recorded $\mathrm{s}-\mathrm{OL} /$ astrocyte $(n=12)$ and $\mathrm{s}-\mathrm{OL} / \mathrm{s}-\mathrm{OL}(n=4)$ pairs. The open red circle indicates the experiment shown in $\mathbf{a}$ and $\mathbf{b}$. Data were fitted by single exponential equations: $\mathrm{s}-\mathrm{OL} /$ astrocyte: $y=165.2 \times \mathrm{e}(-0.27 \times x)+1.46$ (dotted line); s-OL/s-OL: $y=10.76 \times e(-0.249 \times x)+0.435$ (black line). (d) Left: the gap-junction blocker carbenoxolone (CBX) reduced the cross gap-junctional current between s-OLs and astrocytes compared with control conditions. Scale bars, $0.1 \mathrm{mV}$ and $50 \mathrm{~ms}$. Right: summary data showing the inhibition of the gap-junction-mediated current $(n=6$, Wilcoxon signed-rank test, $P=0.03$ ). Data are mean \pm s.e.m. (e) Left: CBX reduced the AP-mediated inward current between neuron-s-OL pairs by $60 \%$. Scale bars, $20 \mathrm{pA}, 40 \mathrm{mV}$ and $20 \mathrm{~ms}$. Right: summary plot showing the reduction of the inward current in s-OLs in the presence of $C B X$ in response to five APs $(n=5)$. Data are mean \pm s.e.m.

$\left[\mathrm{K}^{+}\right]_{\mathrm{o}}$ (Fig. 7e) and a narrow spacing led to plateau depolarisations and seizure-like AP firing in the model cell (Supplementary Fig. 6b). An intercellular distance of $\delta=110 \mathrm{~nm}$ caused, however, a $\left[\mathrm{K}^{+}\right]_{\mathrm{o}}$ increase of $1.2 \mathrm{mM}$ in line with our estimations for the perisomatic extracellular space. Plotting $\left[\mathrm{K}^{+}\right]_{\mathrm{o}}$ and $E_{\mathrm{K}}$ changes during AP firing revealed that reduction of glial Kir conductance density has an impact on the $\mathrm{K}^{+}$driving force (Fig. 7f). With high glial Kir density $\left(0.10 \mathrm{pS} \mu \mathrm{m}^{-2}\right)\left[\mathrm{K}^{+}\right]_{\mathrm{o}}$ accumulated to $8.5 \mathrm{mM}$. In contrast, a 100 -fold reduction of Kir in the glial compartments led to a faster AP-dependent increase in interstitial $\left[\mathrm{K}^{+}\right]_{\mathrm{o}}$ up to a peak concentration of nearly $\sim 10 \mathrm{mM}$, and depolarized $E_{\mathrm{K}}$ by an additional $\sim 5 \mathrm{mV}$ (Fig. $7 \mathrm{f}$ ). As a result, the APs were associated with a reduced afterhyperpolarization, facilitating the generation of additional APs (Fig. 7f). In contrast, for low frequencies, the neuronal AP firing showed no differences between high or low Kir (Fig. 7g). With higher current injections $(>58 \mathrm{pA})$, APs were generated at higher frequencies $(>170 \mathrm{~Hz})$ and reduction of Kir in the glial compartment led to more APs (Fig. 7f,g; Supplementary Fig. 6c). Indeed, when the extracellular volume was increased $(\delta>250 \mathrm{~nm})$, the AP frequency reduced and glial Kir played no role in the initiation of APs (Fig. 7h).

Taken together, the modelling results suggest that placing glia close to the soma membrane causes two opposite changes. On the one hand, a narrower extracellular space increases neuronal excitability by amplifying activity-dependent $\left[\mathrm{K}^{+}\right]_{\mathrm{o}}$. On the other hand, the glia-specific $\mathrm{K}^{+}$buffering limits $\left[\mathrm{K}^{+}\right]_{\mathrm{o}}$ and thereby prevents excessive AP generation. Moreover, glia-specific $\mathrm{K}^{+}$ buffering was in particular noticeable when APs occur at high frequency.

Evidence for modulation of neuronal excitability by s-OLs. To experimentally test whether the perisomatic syncytium around pyramidal neurons impacts neuronal firing, we took advantage of the fact that s-OLs are only found at $\sim 20 \%$ of L5 neurons (Supplementary Fig. 1b). Bright-field and fluorescence inspection in the PLP-ECFP mouse in combination with post hoc labelling of s-OLs confirmed their presence or absence (Fig. 8a; Supplementary Fig. 7a). Assessment of the local three-dimensional arrangement of OLs around these two neuron populations confirmed differences in proximity (with s-OL, $n=13$; without s-OL, $n=8$; Kolmogorov-Smirnoff test $P=0.04$; Fig. 8b). Comparative analysis of the intrinsic properties of L5 neurons with and without s-OL showed that the resting membrane potential of neurons lacking a s-OL $(-76.4 \pm 0.5 \mathrm{mV}, n=17)$ was on average $2.6 \mathrm{mV}$ more depolarized $(-79 \pm 0.4, n=51$, unpaired $t$-test, $P=0.002$ ). Furthermore, the firing properties evoked by a steady depolarizing current step revealed that, while the large majority of neurons with s-OLs were regular firing (86\%, 45 out of 51), L5 neurons without s-OL showed less regular firing $(47 \%, 9$ out of 19 , Fisher's test, $P=0.0007$ ) and instead were more burst firing (53\%, 10 out of 19$)$. Firing behaviour of L5 neurons may be influenced by several factors including composition of intrinsic conductances, the dendritic morphology, as well as the axonal projection targets ${ }^{19,38}$. To isolate the role of extrinsic factors, we grouped L5 neurons according to their firing patterns (Fig. 8c). Analysis of the single APs from burst-firing L5 neurons revealed no difference in amplitude half-width, afterdepolarisation or the rate of rise during the $\mathrm{AP}$ onset (with s-OL $n=6$, w/o s-OL $n=8$, Supplementary Table 2). In addition, analysis of the $F-I$ curve revealed neither a difference in the slope $(P=0.7)$ nor the rheobase $(P=0.11)$ of neurons that fired AP clusters. However, comparison of the first AP cluster revealed that neurons w/o s-OLs $(n=10)$ generated significantly more APs per cluster compared with L5 neurons with s-OL $(n=6$, Mann-Whitney test, $P=0.02$; Fig. 8c). Furthermore, neurons without s-OL were on average firing at higher frequency at a current injection step of $250 \mathrm{pA}$ (two-way analysis of variance (ANOVA), $P=0.0038$; Fig. 8d). In contrast, when the analysis was performed for neurons that were firing at regular low frequency, the presence of s-OLs had no influence on the firing properties (Supplementary Fig. 7b) and comparison of single AP properties revealed no differences (Supplementary Table 2), suggesting that the presence of s-OLs only affects repetitive spike generation.

Finally, the computational model predicted that glial Kir becomes critical when $\left[\mathrm{K}^{+}\right]_{\mathrm{o}}$ reaches higher concentrations, for 

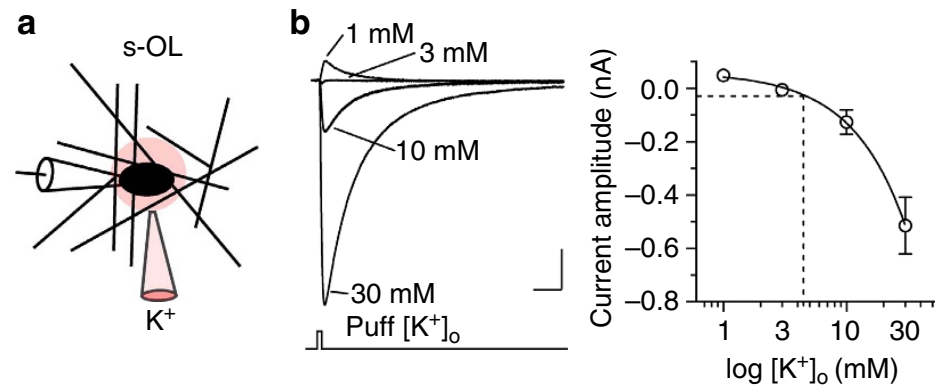

C
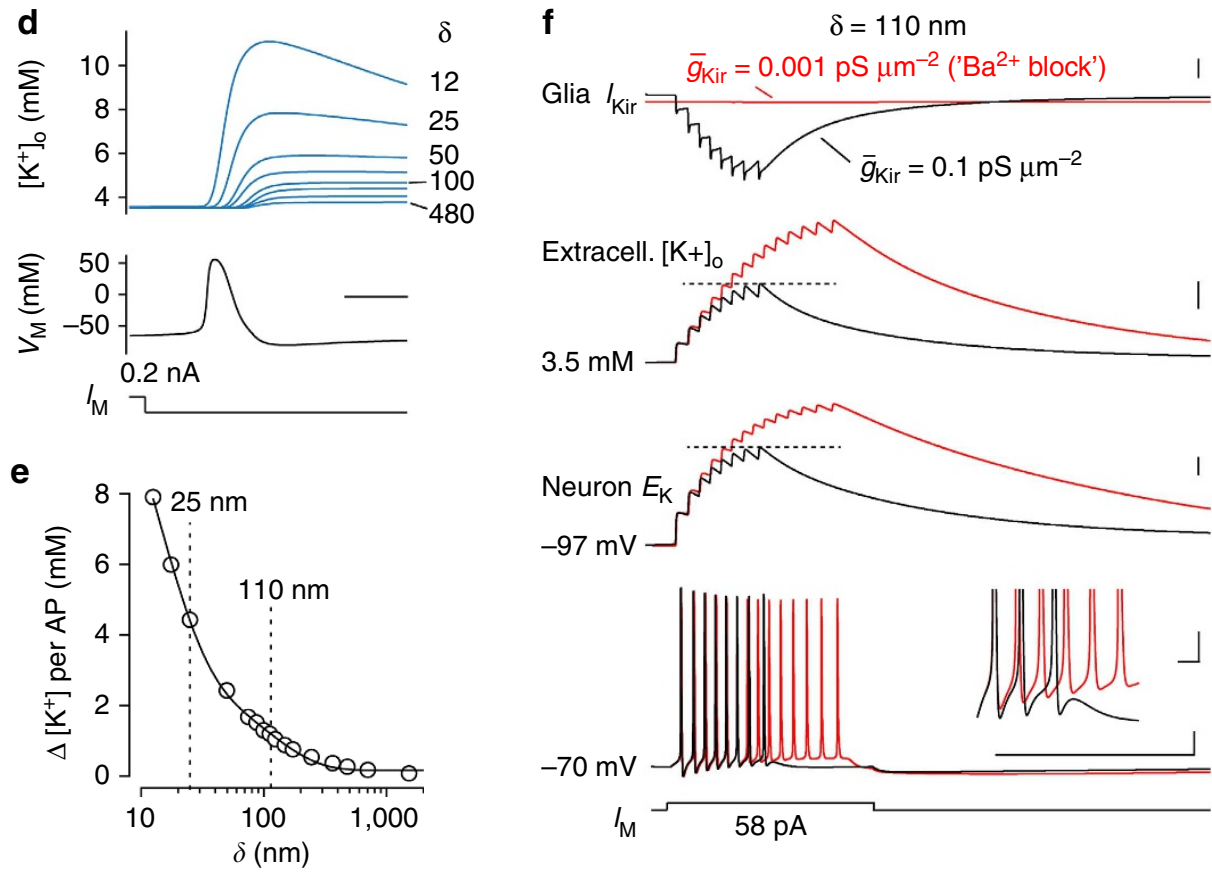
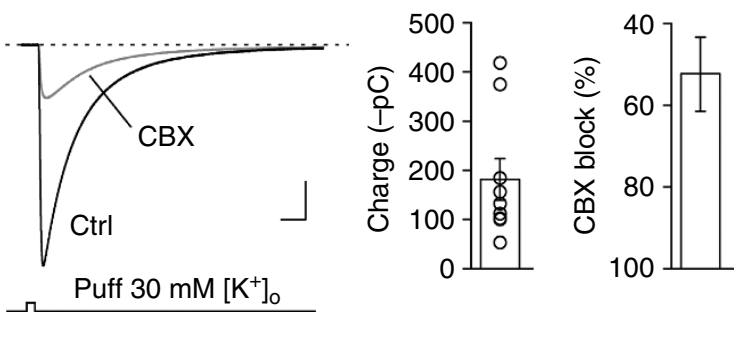

Figure 7 | Glial Kir constraints high-frequency-dependent [K $\left.{ }^{+}\right]_{0}$ elevations. (a) Sketch of experimental [K $\left.{ }^{+}\right]_{0}$ application. (b) Left: average current responses to puffs of various $\left[\mathrm{K}^{+}\right]_{0}$ at a constant holding voltage of $-84 \mathrm{mV}$. Scale bars, $0.1 \mathrm{nA}$ and $0.2 \mathrm{~s}$. Right: Summary plot for all experiments fitted by a linear equation $y=-19.2 x+61.6(1 \mathrm{mM}: n=5 ; 3 \mathrm{mM}: n=6 ; 10 \mathrm{mM}: n=4 ; 30 \mathrm{mM}: n=9)$. Dotted line indicates estimated [K+ $]_{\circ}$ from experimental data of five APs. Data presented as mean \pm s.e.m. (c) Response to local puff application of $30 \mathrm{mM}\left[\mathrm{K}^{+}\right]_{0}$ and inhibition by $100 \mu \mathrm{M}$ CBX. Scale bars, $0.1 \mathrm{nA}$ and $0.2 \mathrm{~s}$. Summary bar graphs for the total charge of the inward current during $30 \mathrm{mM}\left[\mathrm{K}^{+}\right]_{0}$ and percentage of block after application of carbenoxolone $(n=2)$. Data shown as mean \pm s.e.m. (d) Computer simulations of changes in $\left[K^{+}\right]_{0}$ at the soma evoked by a single AP, as a function of the intercellular distance $(\delta)$ between glia and neuron. Scale bar, $1 \mathrm{~ms}$. (e) Summary plot of $\left[\mathrm{K}^{+}\right]_{0}$ change in relation to the intercellular distances between neuron and glia to determine the modelling parameter $(\delta=110 \mathrm{~nm})$. (f) Simulated Kir reduction can generate additional APs. Top to bottom: the Kir current in the glial compartment, extracellular $\left[\mathrm{K}^{+}\right]_{\mathrm{o}}$, neuronal $E_{\mathrm{K}}$ and neuronal membrane potential for high (black) and low (red) conductance densities of Kir. Reducing Kir increases the $\mathrm{K}^{+}$reversal potential. Scale bars, (top to bottom) $20 \mu \mathrm{A} \mathrm{cm}-2,2 \mathrm{mM}, 5 \mathrm{mV}, 100 \mathrm{~ms}$ and $20 \mathrm{mV}$. Inset: the onset of additional APs triggered by impaired Kir (red) shows the reduced fast AP afterhyperpolarization. Scale bars, $5 \mathrm{mV}$ and $5 \mathrm{~ms}$. (g) Input-output function of AP number within the high-frequency cluster versus current injection. Impact of glial Kir reduction only becomes apparent when APs occur at high frequencies ( $>\sim 170 \mathrm{~Hz}$ ) with no impact on low-frequency AP generation (see Supplementary Fig. 6). (h) The positive feedback on AP firing by impaired Kir (closed dots) uptake becomes prominent in narrow interstitial spacing $<100 \mathrm{~nm}$ (fixed current injection of $58 \mathrm{pA}$ ).

example, during repetitive neuronal firing (Fig. $7 \mathrm{~g}$ ). Increased $\left[\mathrm{K}^{+}\right]_{\mathrm{o}}$ can then in turn facilitate the generation of additional APs due to depolarization of $E_{\mathrm{K}}$. To assess the impact of potassium buffering, we recorded from neurons with or w/o s-OL in baseline $(3 \mathrm{mM})$ and elevated $(8 \mathrm{mM})\left[\mathrm{K}^{+}\right]_{\mathrm{o}}$ conditions (Fig. $\left.8 \mathrm{e}\right)$. In $8 \mathrm{mM}$ $\left[\mathrm{K}^{+}\right]_{\mathrm{o}}$, neurons in both the groups showed a strongly depolarized resting membrane potential (with s-OL: $13.5 \pm 1.2 \mathrm{mV}$, without s-OL $14.1 \pm 1.3 \mathrm{mV}, n=7$ cells per group, unpaired $t$-test $P=0.7$ ) and consequently the $F-I$ curve showed a rheobase shift to lower thresholds (Supplementary Fig. 7c). In elevated $\left[\mathrm{K}^{+}\right]_{\mathrm{o}}$, neurons from both the groups generated high-frequency APs (Fig. 8e), which were more numerous in neurons w/o s-OL during current injections of 200 and $250 \mathrm{pA}$ (two-way ANOVA, $P=0.0001, n=7$ cells per group; Fig. 8f,g). Subsequently, on the basis of these results, we calculated the burst probability for the $200 \mathrm{pA}$ current step (Fig. 8h). Consistent with the model predictions of a large role of $\left[\mathrm{K}^{+}\right]_{\mathrm{o}}$ during bursts, the interaction analysis showed that the burst probability was more strongly increased in neurons w/o $\mathrm{s}-\mathrm{OL}$, further supporting our observation that the presence of $\mathrm{s}$-OLs contributes to limit AP generation (repeated measures ANOVA, interaction $P=0.016$; Fig. $8 \mathrm{~h}$ ). In summary, these data suggest that the presence of s-OLs can be interpreted as a proxy for local differences in arrangement of the glial syncytium, which reduces the AP-dependent $\left[\mathrm{K}^{+}\right]_{\mathrm{o}}$ elevations and acts to limit the positive feedback on neuronal excitability.

\section{Discussion}

Satellite oligodendrocytes are ubiquitously found throughout the cortex $^{10,12,17}$ and hippocampus ${ }^{39}$ in rodents and humans ${ }^{14,39,40}$. 

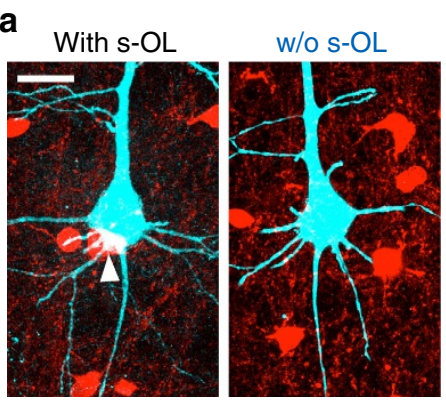

b

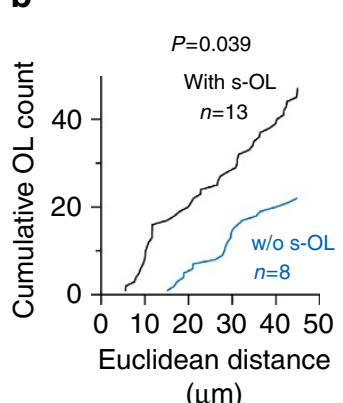

e

With s-OL

Control $\left(3 \mathrm{mM} \mathrm{K}^{+}\right)$
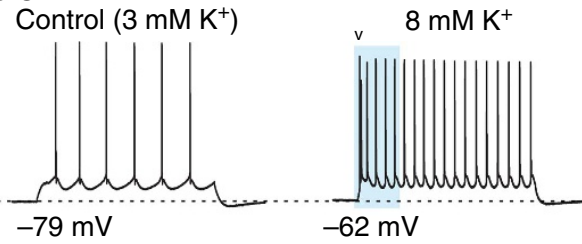

w/o s-OL

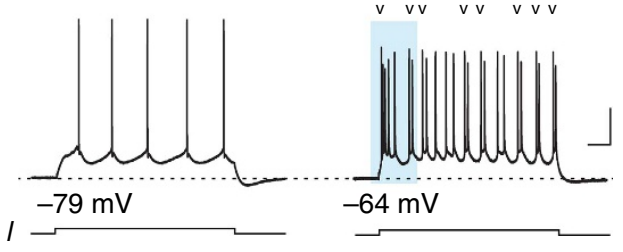

C
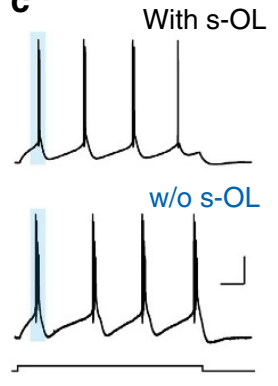

$(\mu \mathrm{m})$
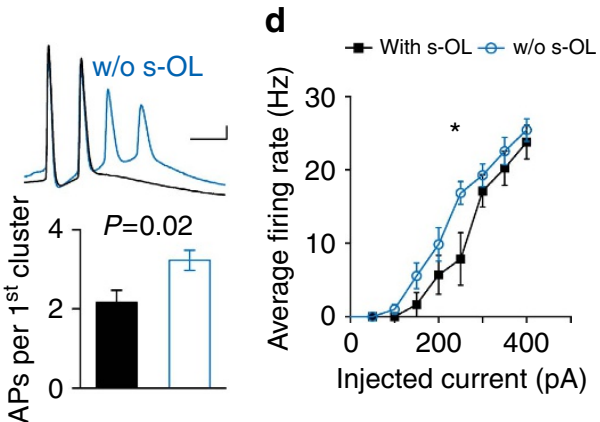

f

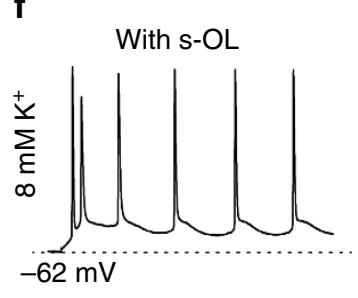

w/o s-OL

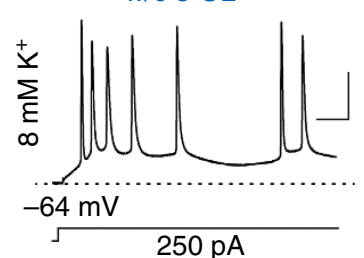

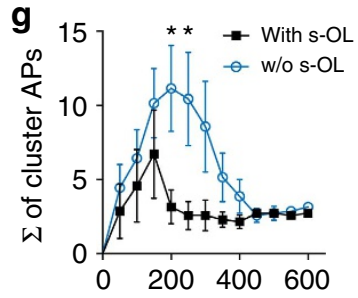

h Injected current $(\mathrm{pA})$

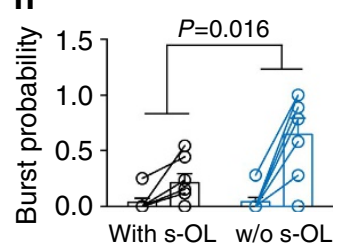

Figure 8 | s-OLs limit high-frequency firing probability. (a) Maximum z-projected images of targeted L5 neurons (cyan) and surrounding oligodendrocytes (red). White arrowhead indicates s-OL. Scale bar, $20 \mu \mathrm{m}$. See also Supplementary Fig. 7a. (b) Distances of oligodendrocytes from the centre of neurons with or without s-OLs show differences in their spatial distances. (c) Left: trains of APs elicited by current injection steps for neurons with and without s-OL. Scale bars, $30 \mathrm{mV}$ and $0.1 \mathrm{~s}$. Top right: high temporal resolution of the first high-frequency AP cluster generated by L5 neurons with or without s-OL. Scale bar, $10 \mathrm{mV}$ and $5 \mathrm{~ms}$. Bottom right: summary data of the average APs per cluster for neurons with $(n=6)$ and without s-OL $(n=10$, Mann-Whitney test, $P=0.02$ ). Data are mean \pm s.e.m. (d) Summary F-I plot of neurons with and without s-OL that generated high-frequency cluster APs. Differences in the average firing rate were observed at $250 \mathrm{pA}$ (two-way ANOVA, $P=0.0038$ ). Data are mean \pm s.e.m. See also Supplementary Fig. $7 \mathrm{~b}$. (e) Left: APs evoked by steady-current injections from neurons with and without s-OL at normal extracellular $\mathrm{K}^{+}$for selected regular firing neurons. Right: after increasing $\left[\mathrm{K}^{+}\right]_{0}$ to $8 \mathrm{mM}$ neurons depolarized and fired more APs with the same current injection. Arrowheads indicate cluster APs. Scale bar, $30 \mathrm{mV}$ and $0.1 \mathrm{~s}$. (f) Increased timescale of the traces in $\mathbf{d}$ in the presence of $8 \mathrm{mM}\left[\mathrm{K}^{+}\right]_{0}$ shows more clustered APs in neurons without a s-OL. Scale bars, $30 \mathrm{mV}$ and $25 \mathrm{~ms}$. (g) Summary plot of the sum of cluster APs for neurons with and without s-OL indicate that the largest difference is observed for current steps between 200 and 300 pA (two-way ANOVA, $P=0.0001, n=7$ cells per group). Data are mean \pm s.e.m. (h) Summary plot displaying burst probability for neurons with and without s-OL in 3 and $8 \mathrm{mM}\left[\mathrm{K}^{+}\right]_{0}$. at a current step of $200 \mathrm{pA}$. Data are single paired experiments and mean \pm s.e.m. Interaction significance was assessed with a two-way ANOVA-repeated measures.

While classified in the literature as non-myelinating similar to $\mathrm{OPCs}^{10,12,14,15}$, we present morphological, ultrastructural and molecular evidence that s-OLs abundantly myelinate axons indistinguishable from type I OLs (Fig. 2) reaffirming their original classification by del Río Hortega $a^{3}$.

What is the functional role of the close apposition of the outer membranes of OLs and neurons? Previous work suggested that s-OLs may provide metabolic support for neurons ${ }^{10}$, protect neurons against apoptosis ${ }^{17}$ or remyelinate axons following injury $^{12}$. Specific arrangements between neurons and glia cells in satellite position, such as the cerebellar Bergmann glia, dynamically regulate extracellular $\mathrm{K}^{+}$, thereby ensuring firing bistability of the Purkinje cell ${ }^{7,41}$. The perisomatic domain of pyramidal layer 5 neurons generates substantial outward $\mathrm{K}^{+}$ current when APs back propagate from the axon into the soma and dendritic tree, and activate fast voltage-gated $\mathrm{K}^{+}$channels ${ }^{24}$. It is well established that buffering of $\left[\mathrm{K}^{+}\right]_{\mathrm{o}}$ and water in the brain is a complex process controlled by passive diffusion, $\mathrm{K}^{+}$ uptake through $\mathrm{Na}^{+} / \mathrm{K}^{+}$pumps and Kir channels as well as spatial $\mathrm{K}^{+}$redistribution via electric coupling of glial cells ${ }^{34,42-45}$. AP-evoked $\mathrm{K}^{+}$release into the extracellular space may be most efficiently controlled when glial syncytia are organized perisomatically and in close contact with the neuronal membrane. Consistent with these features we found that APevoked inward currents in s-OLs were highly sensitive to $\mathrm{Ba}^{2+}$ a Kir channel blocker and partially gap-junction mediated. Importantly, these currents were not different from perisomatic astrocytes that are widely implicated in $\mathrm{K}^{+}$uptake (Figs 3-6; refs $6,34,41,44)$. Based on these results it may be postulated that s-OLs participate in regulation of extracellular $\mathrm{K}^{+}$.

Given the electrical coupling of s-OLs to an astrocyte syncytium what is the role of a satellite OL? The conditional OL-specific knockout of Kir4.1 was characterized by impaired $\mathrm{Ba}^{2+}$-sensitive currents strongly supporting the finding that Kir4.1 is at the membrane in mature OLs (Fig. 4) ${ }^{28,46}$. Unexpectedly, OL Kir4.1 was not required for AP-evoked inward current and setting the resting membrane properties. These results contrast with deletion or mutation of Kir4.1 in astrocytes causing impaired $\mathrm{K}^{+}$siphoning, myelin defects and epilepsy $26,47-49$. There are several possibilities that can account for the lack of detectable impact on $\mathrm{K}^{+}$uptake in Kir4.1-/s-OLs. First, since mature OLs are connected to the astrocyte 
syncytium (Fig. 5) $30-32,50$, the electrical coupling maintains $V_{\mathrm{M}}$ within a glial syncytia even when $\left[\mathrm{K}^{+}\right]_{\mathrm{o}}$ varies. Recent work showed that an $\sim 30 \%$ reduction in the number of astrocytes maintains the isopotentiality of a syncytium ensuring a constant driving force for $\mathrm{K}^{+}$uptake ${ }^{51}$. Indeed, the disruption of OL gapjunctions $\mathrm{Cx} 32$ and $\mathrm{Cx} 47$ have been implicated in deficits of potassium and water siphoning, and myelination defects ${ }^{34}$. Given the dominant role of electrical coupling Kir4.1 activity of a single s-OL may thus be masked by the syncytium. Second, the lack of impact of OL-specific Kir4.1 knockout on the inward current may reflect a differential molecular composition of Kir expression in OLs and astrocytes. OLs express homotetramers of Kir4.1 (ref. 28), a feature which is shared with the endfeet of retinal astrocytes around capillaries ${ }^{52}$. Astrocytes, on the other hand, express heterotetrameric complexes of Kir4.1/Kir5.1 characterized by an approximately fourfold larger single-channel conductance compared with Kir4.1 homotetramers ${ }^{28,53,54}$. Third, it cannot be excluded that OL-specific uptake of $\mathrm{K}^{+}$is complemented by Kir2.1, although this channel is expressed at lower levels compared with Kir4.1 (refs 28,55). Furthermore, a dominant role of astrocytic Kir4.1 in a OL-astrocyte syncytium is supported by the Kir4.1 knockout studies showing not only deficits in potassium buffering and glutamate uptake but also white matter vacuolization ${ }^{26,48,49}$, which could be a consequence of elevated $\left[\mathrm{K}^{+}\right]_{\mathrm{o}}$ leading to myelin degeneration ${ }^{56}$. In line, inward currents of white matter OLs are predominantly carried by $\mathrm{K}^{+}$(ref. 56) supporting an important role for OL physiology. On the basis of these and our present findings, further experiments are required to examine the properties of perisomatic astrocytes and OLs in local AP-evoked $\mathrm{K}^{+}$uptake using the specific conditional astrocytic knockout of Kir4.1 or directly assessing $\left[\mathrm{K}^{+}\right]_{\mathrm{i}}$ by potassium-sensitive dye imaging ${ }^{56}$.

The Nernst equation predicts that depolarization of $E_{\mathrm{K}}$ reduces the driving force for $\mathrm{K}^{+}$, which causes membrane depolarization and increased neuronal excitability. Our computational simulations demonstrated that due to the fast temporal dynamics of Kir-mediated uptake, conducting logarithmically with $\left[\mathrm{K}^{+}\right]_{\mathrm{o}}$ (refs 29,57), $E_{\mathrm{K}}$ is mostly influenced during high-frequency APs (Fig. 7). In good agreement with these predictions, the presence of s-OLs was associated with neuronal excitability changes selectively during high-frequency burst firing and as a function of the baseline $\left[\mathrm{K}^{+}\right]_{\mathrm{o}}$. These findings may suggest that s-OLs have a role in anatomically organizing the perisomatic glial syncytium. This hypothesis remains to be addressed in future studies using the conditional knockout of Kir4.1 in astrocytes or directly visualizing the entire perisomatic glial syncytium. Our results predict that perisomatic syncytia increase the diversity of populations of pyramidal neurons in the neocortex. Since highfrequency burst firing of neocortical pyramidal neurons occurs in vivo during active whisking behaviours and during coincidence detection $^{58,59}$, perisomatic syncytia potentially may contribute to modalities of sensory integration.

In summary, the present work shows that satellite OLs are typical type I OLs and an integral component of an electrically coupled glial syncytium-mediating $\mathrm{K}^{+}$buffering in the perisomatic domain. The results add to an emerging view that by regulating extracellular $\mathrm{K}^{+}$or $\mathrm{Ca}^{2}+$ concentrations glial cells locally shape intrinsic excitability and rhythms in neural circuits $^{4-6,44}$. Neuron-OL-astrocyte interactions may thus not only be critical for driving axonal myelination but also to regulate neuronal excitability on a millisecond timescale.

\section{Methods}

Animals. All procedures involving experimental animals were in agreement with European Union (EU) and national law, complied with guidelines of the Royal Netherlands Academy of Arts and Sciences (KNAW) and were approved by the local animal ethics committee (DEC). Male C57Bl6/J mice were obtained from Harlan or bred in-house. Mice expressing ECFP under the OL-specific PLP promoter (line Q-also PCFQ; here referred to as PLP-ECFP) were obtained from Frank Kirchhoff (University of Saarland) and this line was generated similarly to mice described earlier and expression was specific for OLs ${ }^{60}$. For breeding, PLP-ECFP-positive animals were crossed with C57Bl6/J wild-type mice. Genotyping was performed with a standard PCR protocol (annealing at $60^{\circ} \mathrm{C}$, extension at $70^{\circ} \mathrm{C}$, denaturation at $94^{\circ} \mathrm{C}$ and 35 cycles) and the primers forward: $5^{\prime}$-ATGCGTACCTGACTTTCTCCTTCT- $3^{\prime}$ and reverse: $5^{\prime}$-ACTGGGTGCTCAG GTACTGGTTGT-3', which amplified a 750-bp fragment. For photostimulation experiments, Rbp4-cre_KL100Gsat/Mmucd mice (\#031125-UCD, MMRRC) were crossed with R26-CAG-LSL-2XChETA-tdTomato mice (17455, Jackson Laboratory) to obtain layer 5 specific expression ${ }^{20}$ of the ChR2 variant ChETA. Oligodendrocytic-specific knockout of Kir4.1 was achieved by crossing Kir4.1 1 fl/fl mice $^{26}$ with PLP-cre/ERT mice (\#5975, Jackson Laboratory) and the fluorescent reporter mouse strain B6;129S6-Gt(ROSA)26Sor tm14(CAG-tdTomato)Hze/J (\#7908, Jackson Laboratory). Primers used for genotyping were as described ${ }^{26}$ or as specified by the commercial sources. To induce Cre expression PLP-cre $x$ Kir4.1 ${ }^{\mathrm{fl} / \mathrm{fl}}$ mice were intraperitoneal injected with $75 \mathrm{mg} \mathrm{kg}^{-1}$ tamoxifen (Sigma-Aldrich) dissolved in corn oil for 4 or 5 consecutive days between postnatal days 35-61. Experiments were performed $22 \pm 7$ days $(n=9)$ after start of the injection. Kir4.1 $1^{-1-}$ mice were monitored and did not exhibit abnormal weight changes or developed neurological symptoms as reported for conditional astrocytic or conventional Kir4.1 knockout mice ${ }^{26,48}$. All mice were housed in the institutional animal facilities on a 12-h light/dark cycle with light from 7:00 to 19:00 hours and food (Teklad global diet \#2918, Harlan) and water ad libitum. All experiments were performed with 3-15-week-old (mean $53 \pm 4$ days) mice of both sexes ( $90 \%$ male and $10 \%$ female) with a weight of $22.6 \pm 1.2 \mathrm{~g}$.

Chemicals. Salts for electrophysiology, gabazine, carbenoxolone and biocytin (all Sigma-Aldrich), DAP-5, gabazine, CGP-35348 and CNQX (Tocris) were dissolved in double-distilled water and when feasible stocks of blockers were stored in aliquots at $-20^{\circ} \mathrm{C}$. Blockers were bath applied unless otherwise noted.

Electrophysiology. For electrophysiological recordings, mice were deeply anaesthetized with $3 \%$ isoflurane, decapitated, the brain was quickly removed and placed in ice-cold carbogenated $\left(95 \% \mathrm{O}_{2}, 5 \% \mathrm{CO}_{2}\right)$ cutting solution comprised of (in $\mathrm{mM}$ ) the following: $125 \mathrm{NaCl}, 25 \mathrm{NaHCO}_{3}, 1.25 \mathrm{NaH}_{2} \mathrm{PO}_{4}, 3 \mathrm{KCl}, 25$ glucose, $1 \mathrm{CaCl}_{2}, 6 \mathrm{MgCl}_{2}$ and 1 kynurenic acid. Parasagittal slices of the neocortex were cut at 300- $\mu \mathrm{m}$ thickness (VT1200S, Leica Microsystems, Germany) and incubated at $35^{\circ} \mathrm{C}$ for $35 \mathrm{~min}$ before storing in the same solution at room temperature. Slices were placed in a submerged chamber under an upright microscope (Olympus Nederland BV, Zoeterwoude, The Netherlands) and perfused with heated $\left(33 \pm 2{ }^{\circ} \mathrm{C}\right.$ ) carbogenated artificial cerebrospinal fluid (ACSF) consisting of (in $\mathrm{mM}$ ) the following: $125 \mathrm{NaCl}, 25 \mathrm{NaHCO}_{3}, 1.25 \mathrm{NaH}_{2} \mathrm{PO}_{4}, 3 \mathrm{KCl}, 25$ glucose, $2 \mathrm{CaCl}_{2}$ and $1 \mathrm{MgCl}_{2}$ for recordings. Intracellular solutions for neuronal recordings consisted of (in $\mathrm{mM}$ ) the following: $130 \mathrm{~K}$-gluconate, $10 \mathrm{KCl}, 10 \mathrm{HEPES}$ $4 \mathrm{Mg}$-ATP, $0.3 \mathrm{Na}_{2}$-GTP, $10 \mathrm{Na}_{2}$-phosphocreatine and $\mathrm{pH}$ set to 7.25 ( $\sim 280 \mathrm{mOsm}$ ). For OLs, we routinely used (in $\mathrm{mM}$ ) the following: $130 \mathrm{~K}$-gluconate, $4 \mathrm{NaCl}, 0.5 \mathrm{CaCl}_{2}, 10 \mathrm{EGTA}, 10 \mathrm{HEPES}, 4 \mathrm{Mg}$-ATP, $0.3 \mathrm{Na}_{2}-\mathrm{GTP}, \mathrm{pH}$ set to 7.3 with $\mathrm{KOH}(\sim 280 \mathrm{mOsm})$. In a subset of cells, we additionally included $5 \mathrm{mg} \mathrm{ml}^{-1}$ biocytin. Borosilicate glass pipettes filled with intracellular solution had open pipette resistances of 5-7 M $\Omega$. For identification and morphological analyses, intracellular solutions were supplemented with $200 \mu \mathrm{M}$ Alexa 488 or 75-200 $\mu \mathrm{M}$ Alexa 594 (Life Technologies, USA) dissolved in intracellular solution. This experimental approach allowed identification and distinction of targeted cells, and we could subsequently discern s-OLs, astrocytes and OPCs using live-confocal scans. s-OLs showed multiple internodes of varying lengths, which was in contrast to astrocytes that had a bushy and dense appearance, lacked internodes and were dye coupled with other cells. OPCs exhibited multiple processes of shorter length than s-OLs, were not dye coupled, and lacked bushy processes. The liquid junction potential was corrected for and was calculated (pCLAMP10, Molecular Devices) to be $-15 \mathrm{mV}$ for the neuronal and $-14 \mathrm{mV}$ for the OL intracellular solution. For some experiments, we recorded from OLs using the same intracellular solution as for neurons. The different intracellular solutions did not lead to differences in intrinsic membrane properties of OLs (ANOVA: resting $V_{\mathrm{M}}(P=0.15) ; R_{\mathrm{N}}(P=0.16)$; capacitance $(P=0.68))$ and data were subsequently pooled.

All electrophysiology experiments were controlled and recorded by Axograph X software (v1.3.5, AxoGraph Scientific, Sydney, Australia) and digitized by an AD board (ITC-18, HEKA Elektronik, Lambrecht, Germany). For voltage-clamp recordings, an Axopatch 200B amplifier (Molecular Devices, Sunnyvale, CA, USA) was used. All signals were filtered with the built-in 5 or $10 \mathrm{kHz}$ Bessel filter and sampled between 20 and $50 \mathrm{kHz}$. Series resistance was on average 17.6 $\pm 0.7 \mathrm{M} \Omega$ $(n=100)$ and not compensated for in voltage-clamp recordings. From experiments in which we applied series resistance compensation and prediction by on average $84 \pm 2 \%$ and $\sim 75-100 \%(n=5)$, we observed an increase of current amplitude by $60 \pm 16 \%(n=5)$ but no alterations of the current kinetics $(n=3)$. In currentclamp recordings of neurons and/or glia cells, bridge balance and capacitance compensation were fully applied (BVC 700A amplifier, Dagan Corporation, 
Minneapolis, MN, USA). Some current-clamp recordings of the OLs were made with an Axopatch 200B amplifier using the built-in I-clamp fast mode with bridge balance and capacitance fully compensated. Single APs were evoked by short step current pulses of 3 or $5 \mathrm{~ms}$ adjusted in amplitude to initiate after the stimulus end. Local puffing of solutions was performed with normal patch pipettes connected to a pressure application system (Picospritzer III, Intracel, Herts, UK) with pressure intensity adjusted to the duration of the pulse $(20 \mathrm{~ms})$ and location of the puff (0.1-0.3 bar). The application of different potassium concentrations $(1,3,10$ and $30 \mathrm{mM}$ ) was achieved by adjusting the sodium/potassium ratio of a HEPES-buffered standard ACSF accordingly. For example, for a $10 \mathrm{mM} \mathrm{K}^{+}$ solution, it was composed of (in $\mathrm{mM}$ ) the following: $118 \mathrm{NaCl}, 10 \mathrm{KCl}, 10 \mathrm{HEPES}$, $2 \mathrm{CaCl}_{2}, 1 \mathrm{MgCl}_{2}, 25$ glucose and $\mathrm{pH}$ set to 7.4 with $\mathrm{NaOH}$. In experiments that involved application of $8 \mathrm{mM} \mathrm{K}^{+}$, we added $5 \mathrm{mM} \mathrm{KCl}$ to the standard ACSF. With an intracellular $\left[\mathrm{K}^{+}\right]_{\mathrm{i}}$ of $140 \mathrm{mM}$, this elevation of $\left[\mathrm{K}^{+}\right]_{\mathrm{o}}$ depolarized $E_{\mathrm{k}}$ from -101.4 to $-75.5 \mathrm{mV}\left(33^{\circ} \mathrm{C}\right)$. The calculated change of $E_{\mathrm{Cl}}$ was only about $-1 \mathrm{mV}$. When $8 \mathrm{mM} \mathrm{K}^{+}$was bath-applied experiments were interleaved between neurons with and without s-OL. Experimenter was not blinded for experimental conditions or genotypes.

Analysis of electrophysiological data. Analyses of electrophysiology recordings were performed with Axograph X. $R_{\mathrm{N}}$ was determined by fitting the linear range of voltage responses to current injections ( $\pm 50 \mathrm{pA}$ for L5 neurons and up to $\pm 400 \mathrm{pA}$ for OLs) around the resting membrane potential. The resting membrane potential was determined from traces with zero-current injection or alternatively read from the built-in display after breaking into whole-cell mode (Axopatch $200 \mathrm{~B})$. Resting conductance $(G)$ was calculated from voltage-clamp recordings as $G=\Delta I / V$ were $V$ was a $10 \mathrm{mV}$ hyperpolarizing voltage step from $-70 \mathrm{mV}$ and $\Delta I$ the respective current difference. AP parameters were determined from single-evoked APs or steady-current injections as indicated. The threshold was determined when the slope of the voltage reached $>50 \mathrm{~V} \mathrm{~s}^{-1}$ (refs 35,61). Single APs were only analysed when they initiated with a delay after the end of the current pulse.

Gap-junction coupling of cells was tested by injecting current in one cell and recording the voltage response in both cells; current injections to assess coupling were performed reciprocally. For the analysis, current responses of 10 trials were averaged and the voltage was measured at the end of each current injection step for each cell. The coupling ratio $\left(V_{\text {Receiving }}\right.$ cell $/ V_{\text {Iniected }}$ cell $)$ was calculated for each current step and averaged for all positive and negative current injections.

Subsequently, an average coupling ratio was calculated for each cell pair. The slopes of the F-I curves were estimated from linear fits to either all available data points (regular firing neurons) or to the first data points that were linear (burst-firing neurons). The rheobase was determined as the $x$ interception of the linear fit when $y=0$. Regular and burst-firing neurons were grouped by their ability to generate repetitive APs at high frequencies ${ }^{19}$. Neurons with a short burst of two or more APs $\geq 90 \mathrm{~Hz}$ (cluster) at the onset of the current injection were defined as high-frequency firing neurons and neurons with $>10 \mathrm{~Hz}$ interspike frequencies as regular firing neurons.

Photoactivation of layer 5 neurons. For photostimulation of the $\mathrm{ChR} 2$ variant ChETA in layer 5 neurons, we used a blue light-emitting diode (LED; peak at $470 \mathrm{~nm}$, Thorlabs Inc, Newton NJ, USA) mounted into the light path of the microscope. For the experimental conditions, the LED output power was directly measured in the optical path $(\sim 1.8 \mathrm{~mW})$ with an optical power metre (Newport, Irvine, CA, USA). With this LED intensity, a short light pulse of $3 \mathrm{~ms}$ was sufficient to evoke single APs in layer 5 neurons. To control the area of illumination, a field stop was coupled into the excitation light path.

Live imaging. During electrophysiological recordings, live fluorescence $z$-stacks were obtained with an upright microscope equipped with a confocal scanning unit (Olympus BX61/FV1000, Olympus) and laser lines for 488 and $594 \mathrm{~nm}$ (multi-line argon laser (Showa Optronics, Tokyo, Japan); helium-neon laser (Melles Griot, Carlsbad, CA, USA)). Images were acquired with either $\times 40$ numerical aperture (NA) $0.8, \times 60$ NA 1.0 or $\times 60$ NA 1.1 Olympus water immersion objectives in $z$ step sizes of $1 \mu \mathrm{m}$ (Fluoview software, Olympus).

Immunohistochemistry and image analysis. For post hoc labelling of intracellular dye-filled cells slices were fixed in $4 \%$ paraformaldehyde (PFA) for $10-20 \mathrm{~min}$ before further processing. We used antibodies against MBP (rabbit polyclonal anti-MBP, \#AB980, Millipore or monoclonal mouse anti-MBP,

\#SMI-99P, Covance both 1:250), GFP (ab65556, 1:1,500, Abcam or 75-132, 1:100, Neuromab), Kir4.1 (rabbit polyclonal \#APC-035, 1:300, Alomone, Israel), NeuN (polyclonal guinea-pig, 1:1,000, \#ABN90P, Millipore) and NG2 (polyclonal rabbit, \#AB5320, 1:250, Millipore). Corresponding secondary antibodies were conjugated with Alexa, 488, 555, 594 or 633 (1:500, all Life Technologies) and chosen as suitable. Immunohistochemical labelling was performed as described below. Kir4.1 immunofluorescence labelling was performed on tissue that was fixed for $20 \mathrm{~min}$ in $4 \%$ PFA and subsequently processed identical as described below.

For quantifying the distribution of OLs in neocortical layer 5, we immersionfixed the brains of three mice (C57BL/6, 75 days) in $4 \%$ PFA for $4 \mathrm{~h}$ and cut slices of $40-\mu \mathrm{m}$ thickness on a vibratome (VT1000S, Leica Microsystems, Germany). Sections were labelled with a polyclonal MBP antibody (AB980) of which some isoforms are known to be present in the cytoplasmic domain of OLs ${ }^{62}$ and a NeuN antibody (ABN90P), a pan-neuronal marker, in the presence of $1 \%$ Triton-X 100 at $4{ }^{\circ} \mathrm{C}$ overnight after blocking for $2 \mathrm{~h}$ at room temperature in the same solution without primary antibodies. Secondary antibodies were incubated for $2 \mathrm{~h}$ at room temperature and we used a goat anti guinea-pig-Alexa488 and goat anti rabbit-Alexa555 (Life Technologies). Subsequently, the sections were coversliped with Vectashield with 4,6-diamidino-2-phenylindole (DAPI; Vector Labs). For each animal, eight ROIs from four randomly selected slices in the layer 5 of the somatosensory neocortex were selected and $40 \mu \mathrm{m} z$-stacks were scanned on an inverted confocal microscope with a $\times 40$ PlanApo objective $1.3 \mathrm{NA}$ (Leica SP5, Leica Microsystems) zoom set to 2 and a $z$-step size of $1 \mu \mathrm{m}$ (total volume $\sim 1,501,563 \mu \mathrm{m}^{3}$ ). For the distribution analysis of s-OLs, we first analysed the distribution of all NeuN-positive cells and all cell nuclei that were identified by DAPI. NeuN and DAPI images were $z$-projected, thresholded and neurons, respectively, nuclei manually separated using the line tool. An automated analysis to detect the number of neurons and nuclei was performed by the analyse particles tool with 'exclude on edges' activated (FIJI). Detection of oligodendrocytic cell bodies was performed manually for each $z$-stack. In Adobe Illustrator (Adobe), relative positions of 145 cell bodies were charted to a two-dimensional soma outline obtained from a mouse layer 5 neuron and further processed to create a distribution heatmap ${ }^{63}$. Each OL position was represented as a single point image (brush 5 point and line thickness 4 point), which approximately matched the relative proportions of s-OL cell bodies at L5 neurons. Each position was exported as individual black and white TIFF file, imported into FIJI and converted to binary. Thus, each OL containing pixel was defined as $100 \%$. We then calculated the sum of all images and divided it by the maximum pixel value to obtain the percentage of each pixel to contain a s-OL. A Gaussian blur filter with radius set to 10 was applied and the look-up table (LUT) set. No further adjustment was applied to the images. The two-dimensional cell body area was estimated by outlining the soma area with the polygon selection tool (FIJI) ${ }^{64}$ from distance calibrated average $z$-projected confocal stacks acquired from acute brain slices. All other measurements were performed on either bright-field or confocal images acquired from live tissue. Length measurements were performed on calibrated bright-field or confocal images. The distance between two cells was measured from cell-to-cell border. OL surface area was estimated based on an ellipsoid shape and radii of 5.6, 3.7 and $8.2 \mu \mathrm{m}$ (average values for $n=18$ cells). Internodal length was measured from s-OLs that were individually filled with fluorescence dyes and we could follow single internodes throughout a $z$-stack. We assumed that the internodal length corresponded to the visible internode and measured the length from beginning to end using the segmented line tool. Distance differences between cells in $x y z$ coordinates were converted to Euclidean distance by $d=\sqrt{\left(x^{2}+y^{2}+z^{2}\right)}$

Electron microscopy. To obtain electron microscopy images from single s-OLs, we first identified s-OLs in acute slices of PLP-ECFP mice and filled them with 4-5\% horseradish peroxidase type VI (Sigma-Aldrich) for $>40$ min during wholecell recording. After pipette removal slices containing a single OL were fixed for $\sim 17 \mathrm{~min}$ in freshly prepared $5 \%$ glutaraldehyde in Na-cacodylate (0.1 M, pH 7.4), and then washed in Na-cacodylate buffer and cryoprotected in $25 \%$ sucrose. After saturation slices were embedded in an aluminium boat, frozen on dry ice and resectioned at $40 \mu \mathrm{m}$ with a freezing microtome. For the peroxidase reaction, thin sections were incubated in a Tris- $\mathrm{HCl}$ buffered diaminobenzidine solution containing $0.03 \% \mathrm{H}_{2} \mathrm{O}_{2}$. The diaminobenzidine reaction product was subsequently intensified by a gold-substituted silver peroxidase method ${ }^{65}$. Sections were then rinsed in sodium cacodylate buffer $(0.1 \mathrm{M}, \mathrm{pH} 7.4)$ and post fixed for $20 \mathrm{~min}$ in $1 \% \mathrm{OsO}_{4}$ supplemented with $1 \%$ ferricyanide in cacodylate buffer. Sections were washed, dehydrated and embedded in epoxy resin. Ultrathin sections $(\sim 60 \mathrm{~nm})$ perpendicular to the cortex were made (Ultracut E, Reichert-Jung/Leica), mounted on electron microscopy grids and contrasted with solutions of lead citrate and $0.5 \%$ uranyl acetate (Ultrastain 1 and 2, Laurylab, Brindas, France). Sections were observed with a transmission electron microscope (Tecnai 12, FEI Europe, Eindhoven, Netherlands) and digital images were acquired as TIFF files with a mega view 3 camera (Soft Imaging System/Olympus). The g-ratio for single labelled axons (longitudinally or transversally cut) was calculated from calibrated electron microscopy images as the diameter of the axon divided by the total diameter of the axon including the myelin sheath using FIJI. Major dense lines were counted from images that showed distinct single myelin layers. For antibody labelling of neocortical tissue, freshly prepared $300-\mu \mathrm{m}$ slices were fixed at room temperature for $10 \mathrm{~min}$ in $4 \% \mathrm{PFA}(0.1 \mathrm{M} \mathrm{PB}, \mathrm{pH} 6.5)$ and then in $4 \% \mathrm{PFA}(0.1 \mathrm{M}$ $\mathrm{NaHCO}_{3}$ buffered, $\mathrm{pH} 10.4$ for $\left.10 \mathrm{~min}\right)^{66}$. After rinsing in $\mathrm{PB}(0.1 \mathrm{M}, \mathrm{pH}$ 7.4), the tissue was cryoprotected, frozen and $40-\mu \mathrm{m}$ cryosections were prepared. Sections were then incubated with a rabbit polyclonal antibody against Kir4.1 in PB (1:300, Alomone) for $48 \mathrm{~h}$ at $4{ }^{\circ} \mathrm{C}$. After rinsing, the sections were incubated with a BrightVision Poly-HRP-Goat-Anti-rabbit IgG (Immunologic, Duiven, The Netherlands). Tissues from wild-type and Kir4.1 $1^{-I-}$ animals were processed in parallel. In Kir4.1 $1^{-l-}$ sections, surrounding astrocytic processes exhibited gold particles, indicating successful labelling of the sections (Supplementary Fig. 4a-d). All other steps were performed as described above. 
Potassium concentration estimation in the soma s-OL cleft. To estimate the extracellular $\mathrm{K}^{+}$concentration $\left[\mathrm{K}^{+}\right]_{\mathrm{o}}$ in the cleft region between the $\mathrm{L} 5$ soma and $\mathrm{s}$-OL, we used an analytical solution as a function of cleft height $(\delta)$. Our previous outside-out recordings from rat layer 5 pyramidal neuron somata showed that the $\mathrm{K}^{+}$charge per AP is $\sim 5.3 \mathrm{fC} \mu \mathrm{m}^{-2}$ (ref. 24). The measured spacing between a neuron and s-OL is probably affected by aldehyde fixation ${ }^{67}$ and therefore we use a published value of $\sim 20 \mathrm{~nm}$ (ref. 9). Assuming the cleft height $(\delta)$ is $20 \mathrm{~nm}$ (or $0.020 \mu \mathrm{m}$ ), the $\mathrm{K}^{+}$charge in the extracellular volume may reach 260.5

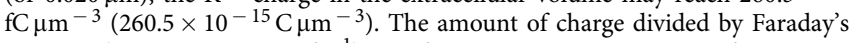
constant $\left(96485.336521 \mathrm{C} \mathrm{mol}^{-1}\right)$ reveals a concentration corresponding to

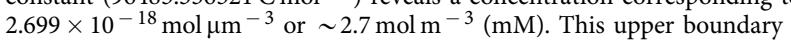
estimation is within range of previous calculations for synaptic clefts in the frog neuromuscular junction, yielding $\Delta\left[\mathrm{K}^{+}\right]_{\mathrm{o}}$ of $2.1 \mathrm{mM}$ per $\mathrm{AP}^{61}$. Taking into account the normal extracellular potassium concentration $(3 \mathrm{mM})$ single APs from the layer 5 soma are expected to cause a peak $\left[\mathrm{K}^{+}\right]_{0}$ within the cleft between the soma and s-OL of $5.7 \mathrm{mM}$. For simplicity, we ignored the lateral and radial $\mathrm{K}^{+}$ diffusion $\left(D=1.96 \times 10^{-9} \mathrm{~m}^{2} \mathrm{~s}^{-1}\right)$ and assumed that all potassium ions will be instantaneously released in the cleft region.

Electrodiffusion NEURON model. To predict the impact of glial Kir4.1 on neuronal AP firing, we used a previous published computational model ${ }^{36,37}$ available at ModelDB (accession ID: 113446). Simulations were performed using the NEURON platform (v7.3). The model consists of a simplified neuron with a somatic compartment and connected with several dendritic compartments containing voltage-gated sodium, potassium, calcium and calcium-activated potassium conductances, as published in detail previously ${ }^{36,37}$. The interstitial space in this model is uniform and implemented as a compartment in which ionic diffusion and buffering takes place as the interstitial space is itself connected with compartments representing glial, segmented with the same spatial resolution as the neuron. Each neuronal segment has a counterpart interstitial and a glial segment in which ionic release, diffusion and uptake, mediated by active mechanisms including Kir, a $3 \mathrm{Na}^{+} / 2 \mathrm{~K}^{+}$exchanger as well as $\mathrm{Ca}^{2+}, \mathrm{Cl}^{-}$and $\mathrm{Na}^{+}$pumps as well as passive diffusion, are calculated. Ion concentration changes are iteratively updated and integrated with the ionic driving forces ${ }^{36,37}$. The glial Kir in this model is based on the mathematical description ${ }^{68}$ where:

$$
\mathrm{g}_{\mathrm{Kir}}=\overline{\mathrm{g}}_{\max } / \sqrt{\left[\mathrm{K}^{+}\right]_{\mathrm{o}}+e \frac{V_{\mathrm{m}}-V_{\mathrm{h}}-E_{\mathrm{k}}}{V_{\mathrm{s}}}}
$$

Here $\overline{\mathrm{g}}_{\max }$ is the maximum conductance at a given $\left[\mathrm{K}^{+}\right]_{\mathrm{o}}, V_{\mathrm{m}}$ is the membrane potential, $V_{\mathrm{h}}$ shifts the opening midpoint potential $-10 \mathrm{mV}$ from $E_{\mathrm{K}}(-97 \mathrm{mV}$ at $\left.\left[\mathrm{K}^{+}\right]_{\mathrm{o}}=3.5 \mathrm{mM}\right)$ and $V_{\mathrm{s}}$ defines the steepness of the voltage dependence. In the mod file, $V_{\mathrm{s}}$ was set to $4 \mathrm{mV}$, representing six elementary charges moving across the entire electric field.

The model was mostly unchanged and run as published at ModelDB ${ }^{36}$, except that spatial segmentation was increased 10 -fold to obtain $3-\mu \mathrm{m}$ segments. To mimic different intercellular distances, we varied $\alpha$, representing the fraction of extracellular volume divided by intracellular volume, between 0.005 and 1.0 (Supplementary Fig. 6c). For clarity, these values were expressed as intercellular distance $(\delta)$ between glia and the soma (with a diameter of $10 \mu \mathrm{m}$ ) and thus ranged between 12 and 2,000 $\mathrm{nm}$. Final simulations were run with an interstitial volume fraction $(\alpha)$ of 0.045 . To mimic glial Kir4.1 reduction, we adjusted Kir between $0.001 \mathrm{pS} \mu \mathrm{m}^{-2}\left(\mathrm{Ba}^{2+}\right.$ block) and $0.10 \mathrm{pS} \mu \mathrm{m}^{-2}$ (control density). Current injections were of short duration and ranged between 0 and $110 \mathrm{pA}$. The temperature in the simulations was nominally set to $33^{\circ} \mathrm{C}$ and simulation time steps $(\mathrm{d} t)$ set to $25 \mu \mathrm{s}$.

Statistics. Data are given as mean \pm s.e.m and the number of experiments and the corresponding statistical test is indicated. Data were statistically analysed with the built-in tools of Prism 6 (GraphPad Software Inc) and SPSS (v.23, IBM Corp, Armonk, NY, USA). When $n \geq 6$, data were tested for normal distribution with a Shapiro-Wilk test and when positive, we applied a paired or unpaired $t$-test subsequently. Not normally distributed data or experiments with $n<6$ were tested with non-parametric Mann-Whitney test when data were unpaired or with Wilcoxon signed-rank test when experiments were paired. Comparisons between more than two groups were performed by a Kruskal-Wallis test or ANOVA where appropriate.

\section{References}

1. Henneberger, C., Papouin, T., Oliet, S. H. R. \& Rusakov, D. A. Long-term potentiation depends on release of D-serine from astrocytes. Nature 463 , 232-236 (2010)

2. Min, R. \& Nevian, T. Astrocyte signaling controls spike timing-dependent depression at neocortical synapses. Nat. Neurosci. 15, 746-753 (2012).

3. Chen, J. et al. Heterosynaptic long-term depression mediated by ATP released from astrocytes. Glia 61, 178-191 (2013).

4. Morquette, P. et al. An astrocyte-dependent mechanism for neuronal rhythmogenesis. Nat. Neurosci. 18, 844-854 (2015).
5. Tong, X. et al. Astrocyte Kir4.1 ion channel deficits contribute to neuronal dysfunction in Huntington's disease model mice. Nat. Neurosci. 17, 694-703 (2014).

6. Wang, F. et al. Astrocytes modulate neural network activity by $\mathrm{Ca}^{2+}$-dependent uptake of extracellular K+. Sci. Signal. 5, ra26 (2012).

7. Vit, J.-P., Ohara, P. T., Bhargava, A., Kelley, K. \& Jasmin, L. Silencing the Kir4.1 potassium channel subunit in satellite glial cells of the rat trigeminal ganglion results in pain-like behavior in the absence of nerve injury. J. Neurosci. 28, 4161-4171 (2008).

8. Baalman, K. et al. Axon initial segment-associated microglia. J. Neurosci. 35, 2283-2292 (2015).

9. Peters, A., Palay, S. L. \& Webster, H. D. The Fine Structure of the Nervous System: Neurons and their Supporting Cells 3 (Oxford Univ. Press, 1991).

10. Takasaki, C. et al. Cytochemical and cytological properties of perineuronal oligodendrocytes in the mouse cortex. Eur. J. Neurosci. 32, 1326-1336 (2010).

11. Kruger, L. \& Maxwell, D. S. Electron microscopy of oligodendrocytes in normal rat cerebrum. Am. J. Anat. 118, 411-435 (1966).

12. Ludwin, S. K. The perineuronal satellite oligodendrocyte. a role in remyelination. Acta Neuropathol. 47, 49-53 (1979).

13. del Río Hortega, P. Tercera aportación al conocimiento morfológico e interpretación funcional de la oligodendroglía. Mem. Real Soc. Esp. Hist. Nat. 14, 5-122 (1928).

14. Szuchet, S. et al. The genetic signature of perineuronal oligodendrocytes reveals their unique phenotype. Eur. J. Neurosci. 34, 1906-1922 (2011).

15. Butt, A. M. in Neuroglia. (eds Kettenmann, H. \& Ransom, B. R.) 62-73 (Oxford Univ. Press, 2013).

16. Nave, K.-A. Myelination and support of axonal integrity by glia. Nature $\mathbf{4 6 8}$, 244-252 (2010).

17. Taniike, M. et al. Perineuronal oligodendrocytes protect against neuronal apoptosis through the production of lipocalin-type prostaglandin $\mathrm{D}$ synthase in a genetic demyelinating model. J. Neurosci. 22, 4885-4896 (2002).

18. Kole, M. H. P. \& Stuart, G. J. Signal processing in the axon initial segment. Neuron 73, 235-247 (2012).

19. Kole, M. H. P. First node of Ranvier facilitates high-frequency burst encoding. Neuron 71, 671-682 (2011).

20. Beltramo, R. et al. Layer-specific excitatory circuits differentially control recurrent network dynamics in the neocortex. Nat. Neurosci. 16, 227-234 (2013).

21. Bergles, D. E., Roberts, J. D., Somogyi, P. \& Jahr, C. E. Glutamatergic synapses on oligodendrocyte precursor cells in the hippocampus. Nature 405, 187-191 (2000).

22. Káradóttir, R., Cavelier, P., Bergersen, L. H. \& Attwell, D. NMDA receptors are expressed in oligodendrocytes and activated in ischaemia. Nature 438, 1162-1166 (2005).

23. Gipson, K. \& Bordey, A. Analysis of the $\mathrm{K}+$ current profile of mature rat oligodendrocytes in situ. J. Membr. Biol. 189, 201-212 (2002).

24. Hallermann, S., de Kock, C. P. J., Stuart, G. J. \& Kole, M. H. P. State and location dependence of action potential metabolic cost in cortical pyramidal neurons. Nat. Neurosci. 15, 1007-1014 (2012).

25. Maldonado, P. P., Vélez-Fort, M., Levavasseur, F. \& Angulo, M. C. Oligodendrocyte precursor cells are accurate sensors of local $\mathrm{K}+$ in mature gray matter. J. Neurosci. 33, 2432-2442 (2013).

26. Djukic, B., Casper, K. B., Philpot, B. D., Chin, L.-S. \& McCarthy, K. D. Conditional knock-out of Kir4.1 leads to glial membrane depolarization, inhibition of potassium and glutamate uptake, and enhanced short-term synaptic potentiation. J. Neurosci. 27, 11354-11365 (2007).

27. Kalsi, A. S., Greenwood, K., Wilkin, G. \& Butt, A. M. Kir4.1 expression by astrocytes and oligodendrocytes in CNS white matter: a developmental study in the rat optic nerve. J. Anat. 204, 475-485 (2004).

28. Schirmer, L. et al. Differential loss of KIR4.1 immunoreactivity in multiple sclerosis lesions. Ann. Neurol. 75, 810-828 (2014).

29. Hibino, H. et al. Inwardly rectifying potassium channels: their structure, function, and physiological roles. Physiol. Rev. 90, 291-366 (2010).

30. Tress, O. et al. Panglial gap junctional communication is essential for maintenance of myelin in the CNS. J. Neurosci. 32, 7499-7518 (2012).

31. Wasseff, S. K. \& Scherer, S. S. Cx32 and Cx47 mediate oligodendrocyte:astrocyte and oligodendrocyte:oligodendrocyte gap junction coupling. Neurobiol. Dis. 42, 506-513 (2011).

32. Maglione, M. et al. Oligodendrocytes in mouse corpus callosum are coupled via gap junction channels formed by connexin 47 and connexin32. Glia 58, 1104-1117 (2010).

33. Ransom, B. R. \& Kettenmann, H. Electrical coupling, without dye coupling, between mammalian astrocytes and oligodendrocytes in cell culture. Glia $\mathbf{3}$, 258-266 (1990).

34. Menichella, D. M. et al. Genetic and physiological evidence that oligodendrocyte gap junctions contribute to spatial buffering of potassium released during neuronal activity. J. Neurosci. 26, 10984-10991 (2006). 
35. Kole, M. H. P. \& Stuart, G. J. Is action potential threshold lowest in the axon? Nat. Neurosci. 11, 1253-1255 (2008).

36. Somjen, G. G., Kager, H. \& Wadman, W. J. Computer simulations of neuronglia interactions mediated by ion flux. J. Comput. Neurosci. 25, 349-365 (2008).

37. Kager, H., Wadman, W. J. \& Somjen, G. G. Seizure-like afterdischarges simulated in a model neuron. J. Comput. Neurosci. 22, 105-128 (2007).

38. Hattox, A. M. \& Nelson, S. B. Layer V neurons in mouse cortex projecting to different targets have distinct physiological properties. J. Neurophysiol. $\mathbf{9 8}$, 3330-3340 (2007).

39. van Landeghem, F. K. H., Weiss, T. \& von Deimling, A. Expression of PACAP and glutamate transporter proteins in satellite oligodendrocytes of the human CNS. Regul. Pept. 142, 52-59 (2007).

40. Vostrikov, V. M., Uranova, N. A. \& Orlovskaya, D. D. Deficit of perineuronal oligodendrocytes in the prefrontal cortex in schizophrenia and mood disorders. Schizophr. Res. 94, 273-280 (2007).

41. Wang, F., Xu, Q., Wang, W., Takano, T. \& Nedergaard, M. Bergmann glia modulate cerebellar Purkinje cell bistability via $\mathrm{Ca} 2+$-dependent $\mathrm{K}+$ uptake. Proc. Natl Acad. Sci. USA 109, 7911-7916 (2012).

42. Kofuji, P. \& Newman, E. A. Potassium buffering in the central nervous system. Neuroscience 129, 1045-1056 (2004).

43. Holthoff, K. \& Witte, O. W. Directed spatial potassium redistribution in rat neocortex. Glia 29, 288-292 (2000).

44. Pannasch, U., Derangeon, M., Chever, O. \& Rouach, N. Astroglial gap junctions shape neuronal network activity. Commun. Integr. Biol. 5, 248-254 (2012).

45. Syková, E. \& Nicholson, C. Diffusion in brain extracellular space. Physiol. Rev. 88, 1277-1340 (2008).

46. Poopalasundaram, S. et al. Glial heterogeneity in expression of the inwardly rectifying $\mathrm{K}(+)$ channel, Kir4.1, in adult rat CNS. Glia 30, 362-372 (2000).

47. Bockenhauer, D. et al. Epilepsy, ataxia, sensorineural deafness, tubulopathy, and KCNJ10 mutations. N. Engl. J. Med. 360, 1960-1970 (2009).

48. Neusch, C., Rozengurt, N., Jacobs, R. E., Lester, H. A. \& Kofuji, P. Kir4.1 potassium channel subunit is crucial for oligodendrocyte development and in vivo myelination. J. Neurosci. 21, 5429-5438 (2001)

49. Haj-Yasein, N. N. et al. Evidence that compromised K+ spatial buffering contributes to the epileptogenic effect of mutations in the human Kir4.1 gene (KCNJ10). Glia 59, 1635-1642 (2011).

50. Griemsmann, S. et al. Characterization of panglial gap junction networks in the thalamus, neocortex, and hippocampus reveals a unique population of glial cells. Cereb. Cortex 25, 3420-3433 (2014).

51. Ma, B. et al. Gap junction coupling confers isopotentiality on astrocyte syncytium. Glia 64, 214-226 (2016).

52. Kofuji, P. et al. Kir potassium channel subunit expression in retinal glial cells: implications for spatial potassium buffering. Glia 39, 292-303 (2002).

53. Pessia, M., Tucker, S. J., Lee, K., Bond, C. T. \& Adelman, J. P. Subunit positional effects revealed by novel heteromeric inwardly rectifying $\mathrm{K}+$ channels. $E M B O$ J. 15, 2980-2987 (1996).

54. Tanemoto, M., Kittaka, N., Inanobe, A. \& Kurachi, Y. In vivo formation of a proton-sensitive $\mathrm{K}+$ channel by heteromeric subunit assembly of Kir5.1 with Kir4.1. J. Physiol. 525(Pt 3): 587-592 (2000).

55. Zhang, Y. et al. An RNA-sequencing transcriptome and splicing database of glia, neurons, and vascular cells of the cerebral cortex. J. Neurosci. 34, 11929-11947 (2014).

56. Hamilton, N. B., Kolodziejczyk, K., Kougioumtzidou, E. \& Attwell, D. Proton-gated $\mathrm{Ca}(2+)$-permeable TRP channels damage myelin in conditions mimicking ischaemia. Nature 529, 523-527 (2016).

57. Périer, F., Radeke, C. M. \& Vandenberg, C. A. Primary structure and characterization of a small-conductance inwardly rectifying potassium channel from human hippocampus. Proc. Natl Acad. Sci. USA 91, 6240-6244 (1994).

58. Xu, N.-L. et al. Nonlinear dendritic integration of sensory and motor input during an active sensing task. Nature 492, 247-251 (2012).

59. Larkum, M. A cellular mechanism for cortical associations: an organizing principle for the cerebral cortex. Trends Neurosci. 36, 141-151 (2013).
60. Hirrlinger, P. G. et al. Expression of reef coral fluorescent proteins in the central nervous system of transgenic mice. Mol. Cell. Neurosci. 30, 291-303 (2005).

61. Attwell, D. \& Iles, J. F. Synaptic transmission: ion concentration changes in the synaptic cleft. Proc. Natl Acad. Sci. USA 206, 115-131 (1979).

62. Hardy, R. J., Lazzarini, R. A., Colman, D. R. \& Friedrich, V. L. Cytoplasmic and nuclear localization of myelin basic proteins reveals heterogeneity among oligodendrocytes. J. Neurosci. Res. 46, 246-257 (1996).

63. Bortone, D. S., Olsen, S. R. \& Scanziani, M. Translaminar inhibitory cells recruited by layer 6 corticothalamic neurons suppress visual cortex. Neuron $\mathbf{8 2}$, 474-485 (2014).

64. Schindelin, J. et al. Fiji: an open-source platform for biological-image analysis. Nat. Methods 9, 676-682 (2012).

65. van den Pol, A. N. \& Gorcs, T. Synaptic relationships between neurons containing vasopressin, gastrin-releasing peptide, vasoactive intestinal polypeptide, and glutamate decarboxylase immunoreactivity in the suprachiasmatic nucleus: dual ultrastructural immunocytochemistry with gold-substituted silver peroxidase. J. Comp. Neurol. 252, 507-521 (1986).

66. Eldred, W. D., Zucker, C., Karten, H. J. \& Yazulla, S. Comparison of fixation and penetration enhancement techniques for use in ultrastructural immunocytochemistry. J. Histochem. Cytochem. 31, 285-292 (1983).

67. Korogod, N., Petersen, C. C. H. \& Knott, G. W. Ultrastructural analysis of adult mouse neocortex comparing aldehyde perfusion with cryo fixation. Elife 4, e05793 (2015).

68. van Heukelom, J. S. The role of the potassium inward rectifier in defining cell membrane potentials in low potassium media, analysed by computer simulation. Biophys. Chem. 50, 345-360 (1994).

\section{Acknowledgements}

We thank Bob Oudejans for data acquisition and analysis and Sharon de Vries for excellent technical assistance. Tycho Hoogland, Paloma Maldonado, Mustafa Hamada and Marko Popovic provided helpful discussion and critical reading of earlier versions of the manuscript. We furthermore thank Frank Kirchhoff for providing the ECFP mouseline and Erlend A Nagelhus for providing the Kir4.1 ${ }^{\mathrm{fl}}$ mouseline. We are thankful for the Electron Microscopy Centre Amsterdam (EMCA) of the Academic Medical Centre providing electron microscopy support. This research was supported by the European Research Council (FP7/2007-2013)/ERC Grant agreement P261114 and the Hersenstichting Nederland (Grant \#2013(1)-160).

\section{Author contributions}

Conceptualization of the study by M.K. Design of the study and experiments A.B. and M.K. Performance of the experiments, analysis of the data and writing of the manuscript A.B., J.K. and M.K.

\section{Additional information}

Supplementary Information accompanies this paper at http://www.nature.com/ naturecommunications

Competing financial interests: The authors declare no competing financial interests.

Reprints and permission information is available online at http://npg.nature.com/ reprintsandpermissions/

How to cite this article: Battefeld, A. et al. Myelinating satellite oligodendrocytes are integrated in a glial syncytium constraining neuronal high-frequency activity. Nat. Commun. 7:11298 doi: 10.1038/ncomms11298 (2016).

This work is licensed under a Creative Commons Attribution 4.0 International License. The images or other third party material in this article are included in the article's Creative Commons license, unless indicated otherwise in the credit line; if the material is not included under the Creative Commons license, users will need to obtain permission from the license holder to reproduce the material. To view a copy of this license, visit http://creativecommons.org/licenses/by/4.0/ 